\title{
Stabilization of the cardiac sarcolemma by sarcospan rescues DMD-associated cardiomyopathy
}

\author{
Michelle S. Parvatiyar, ${ }^{1,2}$ Alexandra J. Brownstein, ${ }^{1,2}$ Rosemeire M. Kanashiro-Takeuchi, ${ }^{3,4}$ \\ Judd R. Collado, ${ }^{1}$ Karissa M. Dieseldorff Jones, ${ }^{5}$ Jay Gopal, ${ }^{1}$ Katherine G. Hammond, ${ }^{1,2}$ \\ Jamie L. Marshall,, ${ }^{1,2}$ Abel Ferrel, ${ }^{1}$ Aaron M. Beedle, ${ }^{6}$ Jeffrey S. Chamberlain, ${ }^{7}$ Jose Renato Pinto, ${ }^{5}$ \\ and Rachelle H. Crosbie $e^{1,2,8,9}$ \\ 'Department of Integrative Biology \& Physiology and 2Center for Duchenne Muscular Dystrophy, UCLA, Los Angeles, \\ California, USA. ${ }^{3}$ nterdisciplinary Stem Cell Institute, University of Miami, Florida, USA. ${ }^{4}$ Department of Molecular and \\ Cellular Pharmacology, University of Miami Miller School of Medicine, Miami, Florida, USA. ${ }^{5}$ Department of Biomedical \\ Sciences, College of Medicine, Florida State University, Tallahassee, Florida, USA. ${ }^{6}$ Department of Pharmaceutical \\ Sciences, Binghamton University State University of New York, Binghamton, New York, USA. 'Department of Neurology, \\ University of Washington, Seattle, Washington, USA. ${ }^{8}$ Department of Neurology, David Geffen School of Medicine at \\ UCLA, Los Angeles, California, USA. ${ }^{9}$ Molecular Biology Institute, UCLA, Los Angeles, California, USA.
}

In the current preclinical study, we demonstrate the therapeutic potential of sarcospan (SSPN) overexpression to alleviate cardiomyopathy associated with Duchenne muscular dystrophy (DMD) utilizing dystrophin-deficient $m d x$ mice with utrophin haploinsufficiency that more accurately represent the severe disease course of human DMD. SSPN interacts with dystrophin, the DMD disease gene product, and its autosomal paralog utrophin, which is upregulated in DMD as a partial compensatory mechanism. SSPN-Tg mice have enhanced abundance of fully glycosylated $\alpha$-dystroglycan, which may further protect dystrophin-deficient cardiac membranes. Baseline echocardiography revealed that SSPN improves systolic function and hypertrophic indices in $\mathbf{m d x}$ and $m d x$ :utr-heterozygous mice. Assessment of SSPN-Tg $m d x$ mice by hemodynamic pressurevolume methods highlighted enhanced systolic performance compared with $\mathbf{m d x}$ controls. SSPN restored cardiac sarcolemma stability, the primary defect in DMD disease; reduced fibrotic response; and improved contractile function. We demonstrate that SSPN ameliorated more advanced cardiac disease in the context of diminished sarcolemma expression of utrophin and $\beta 1 D$ integrin, which mitigate disease severity, and partially restored responsiveness to $\beta$-adrenergic stimulation. Overall, our current and previous findings suggest that SSPN overexpression in DMD mouse models positively affects skeletal, pulmonary, and cardiac performance by addressing the stability of proteins at the sarcolemma that protect the heart from injury, supporting SSPN and membrane stabilization as a therapeutic target for DMD.

Authorship note: AJB and RMKT contributed equally as co-second authors.

Conflict of interest: The authors have declared that no conflict of interest exists.

Copyright: (c) 2019 American Society for Clinical Investigation

Submitted: August 6, 2018

Accepted: April 23, 2019

Published: June 6, 2019.

Reference information: JCl Insight. 2019;4(11):e123855. https://doi. org/10.1172/jci.insight.123855.

\section{Introduction}

Duchenne muscular dystrophy (DMD) is a lethal childhood neuromuscular disorder that results in progressive muscle weakness, respiratory difficulties, and cardiovascular dysfunction that dramatically shortens life span. DMD is the most common lethal genetic disorder in children and affects 1 in approximately 5500 males $(1,2)$. Mutations in the X-linked dystrophin gene DYS1 cause loss of the functional protein, along with loss of the entire dystrophin-glycoprotein complex (DGC) from the sarcolemma. In $\mathrm{DMD}$, dystrophin protein is degraded $(3,4)$ and/or not properly transported to the membrane (5). In Becker muscular dystrophy, in-frame deletion mutations produce a less functional protein that localizes to the cell membrane. X-linked dilated cardiomyopathy manifests only in the heart and is caused by specific mutations located in the N-terminus of the dystrophin gene (6). Individuals with DMD develop dilated cardiomyopathy by their second decade of life. The typical disease course of DMD-associated cardiomyopathy initially manifests as ECG abnormalities, development of diastolic dysfunction, fibrosis detected by MRI, dilatation of cardiac cavities, and systolic dysfunction followed by end-stage heart 
failure (7). Improvements in respiratory care have extended the life span of DMD patients, increasing the likelihood that patients will develop cardiomyopathy. Management of patient cardiac symptoms largely includes angiotensin converting enzyme inhibitors, steroids and beta blockers that delay the onset of cardiac dysfunction, and development of adverse remodeling.

Many therapeutic platforms are being developed to combat DMD disease: exon-skipping strategies, gene editing, and development of adenoviral deliverable miniaturized utrophin and dystrophin constructs as well as membrane stabilizers. In 2016, the FDA accelerated approval of the first DMD-specific drug eteplirsen (Exondys 51), an exon-skipping therapeutic that promotes the production of functional dystrophin (8). However, eteplirsen only targets $13 \%$ of the DMD population, and current formulations and delivery strategies do not effectively target the heart. The current study focuses on investigating the therapeutic potential of sarcospan (SSPN), which associates with dystrophin and contributes to normal cardiac function. We sought to determine whether therapeutic approaches that benefit dystrophic skeletal muscle are also efficacious for the treatment of DMD cardiomyopathy $(9,10)$. Findings from our DMD studies in mice have elucidated additional roles of structural proteins that also provide cell membrane support.

Dystrophin is a member of the DGC consisting of integral and peripheral membrane proteins (10, 11). Dystrophin is located on the cytoplasmic face of the sarcolemma and connects intracellular actin with $\beta$-dystroglycan ( $\beta$-DG) of the DGC complex $(11,12)$. $\beta$-DG is complexed with $\alpha$-DG, which is heavily glycosylated in its mucin domain with $O$-linked glycans that bind to laminin in the extracellular matrix (13). Also associated with the complex are 4 sarcoglycan proteins ( $\alpha$-SG, $\beta$-SG, $\delta$-SG, $\gamma$-SG) that contain a single transmembrane domain. The protein SSPN (14) contains 4 transmembrane domains and tightly associates with the SGs $(15,16)$ and $\beta$-DG (17). Other laminin-binding complexes are also situated at the cell membrane, including the $\alpha 7 / \beta 1 \mathrm{D}$ integrin complex and the utrophin-glycoprotein complex (UGC), largely similar to the DGC with the exception that utrophin is substituted for dystrophin and shares $80 \%$ sequence homology with dystrophin (18). The UGC is localized at neuromuscular junctions in skeletal muscle (19); however, it colocalizes with dystrophin at the cardiomyocyte sarcolemma (19). Utrophin exhibits distinctive localization from dystrophin in cardiac muscle, as it is additionally expressed in Purkinje fibers and is localized to intercalated discs (20). In dystrophic $m d x$ mice (the genetic DMD mouse model), utrophin is upregulated in skeletal muscle and assumes a sarcolemmal distribution as a compensatory measure to protect the dystrophin-devoid sarcolemma from contraction-induced injury (21). Humans exhibit more severe DMD disease, which is postulated in part to result from a diminished ability to upregulate utrophin to compensate for dystrophin deficiency. During development, utrophin is expressed at high levels at the sarcolemma and is able to function in place of dystrophin $(22,23)$. A number of efforts have been directed toward the development of drug-based utrophin therapies that can reactivate the fetal isoform utrophin-A in DMD patients based on amelioration of dystrophic symptoms in $m d x$ mice engineered to overexpress utrophin (24-26). The Phase II PhaseOut DMD trial (NCT02858362), testing the efficacy of the utrophin-upregulating compound ezutromid in ambulatory DMD boys, was discontinued after failing to show efficacy.

Utrophin deficiency has been shown to exacerbate cardiac dysfunction in $m d x$ mice. Studies performed with double-knockout (dko) mice lacking both dystrophin and utrophin exhibit similar courses of dilated cardiomyopathy compared with $m d x$ mice $(27,28)$. However, the timeframe for developing cardiac function is much shorter, and by 20 weeks the dko mice died early $(27,28)$. To assess the dependence of SSPN rescue on robust utrophin upregulation, we utilized $m d x$ :utr-heterozygous ( $m d x$ :utr-het) mice, the most relevant mouse model available for DMD cardiac studies. These mice offer a more severe manifestation of cardiac disease that develops over a much shorter time period and allow testing of whether SSPN addresses sarcolemma instability in a more DMD relevant context (29). This experimental design allows us to examine SSPN overexpression as an alternative method for induction of utrophin expression in DMD hearts and as a strategy to ameliorate cardiac dysfunction.

Our studies exploring the therapeutic benefit of SSPN-based sarcolemma stabilization of DMD skeletal and cardiac muscle have revealed that SSPN overexpression is effective at improving numerous aspects of DMD pathology $(5,30,31)$. Even low levels of SSPN overexpression can enhance $m d x$ cardiac membrane stability, upregulate utrophin expression, and improve cardiac function (30), in addition to addressing skeletal muscle pathology and contraction-induced injury to myofibers (5, 31-34). Furthermore, we have demonstrated effective transduction of the heart using adeno-associated virus type 6-expressing SSPN (AAV6-SSPN). AAV6-SSPN effectively delivers this small 25-kDa protein, therefore establishing, its 
suitability for gene therapy approaches. The current study addresses the mechanistic basis of SSPN rescue of cardiac pathology in several relevant genotypic and phenotypic DMD mouse models and serves as a preclinical study, assessing the effectiveness of SSPN in upregulating utrophin and UGC-associated proteins in human DMD disease in order to preserve cardiac muscle integrity and contractility.

\section{Results}

SSPN overexpression improves cardiac histology in DMD mouse models. In order to investigate the effect of SSPN on DMD-associated cardiomyopathy, we evaluated $m d x$ and utrophin haploinsufficient $m d x\left(m d x: \mathrm{utr}^{+/-}\right)$mice that overexpressed Tg SSPN. Widespread SSPN-Tg overexpression in cardiac cross sections from the DMD mouse model was revealed by SSPN antibody detection using indirect immunofluorescence (Figure 1A). The staining detected was reflective of both endogenous and Tg SSPN, as the Tg encodes murine SSPN. SSPN was significantly reduced from the sarcolemma when dystrophin was absent $(m d x)$ and was even further reduced in dystrophin-deficient hearts with reduced utrophin expression ( $\left.m d x: u_{t r}^{+/-}\right)$. The overexpression of SSPN resulted in increased levels of SSPN protein at the sarcolemma of $m d x$ and $m d x$ :utr-het hearts. To assess the relative quantity of SSPN protein at the sarcolemma of the DMD SSPN-Tg hearts, we quantified immunofluorescence images to report the total corrected fluorescence. SSPN was robustly overexpressed in both $m d x$ and $m d x$ :utr-het hearts, although the relative levels were greater in $m d x$ relative to $m d x$ : utr-het hearts (Figure 1E). To determine whether SSPN overexpression improved DMD cardiac tissue pathology, Masson's trichrome, a marker for collagen, was used to determine its effects on fibrosis (Figure 1, B and C). It was anticipated that stabilization of the DMD sarcolemma by SSPN overexpression would lessen downstream pathological changes induced by membrane fragility and thereby protect from cardiomyocyte loss and fibrotic deposition. Compared with WT hearts, $m d x$ hearts displayed a significant increase in fibrotic area. The $m d x^{\mathrm{TG}}$ tissue sections exhibited a substantial, although not significant, reduction in fibrosis, quantified as the percentage of total area when compared with $m d x$ controls, and these results were intermediate to WT values (Figure 1D). Fibrosis in the more severe DMD $m d x$ :utr-het model overexpressing SSPN-Tg trended toward improvement relative to $m d x$ :utr-het controls (Figure $1, \mathrm{~B}-\mathrm{D}$ ). The organization of $m d x^{\mathrm{TG}}$ tissue appeared similar to WT tissue, with the exception of a few localized fibrotic regions present, and, similarly, $m d x$ :utr-het ${ }^{\mathrm{TG}}$ hearts exhibited overall smaller fibrotic areas when compared with $m d x$ :utr-het cardiac tissue (Figure 1, B and C). To determine the potential of SSPN overexpression to alleviate DMD cardiomyopathy, pilot studies were conducted to assess the therapeutic potential of AAV6-SSPN to increase SSPN expression in the heart. To visualize SSPN localization in the heart, transverse whole heart sections of the $m d x+$ AAV6-SSPN-treated mice were stained with SSPN antibody. The high level of SSPN expression in these hearts demonstrated that AAV6-SSPN could be effectively delivered to and expressed in the $m d x$ myocardium (Figure $1 \mathrm{~F}$ ).

SSPN overexpression improves baseline cardiac parameters in DMD mouse models. To assess whether the mechanism of SSPN rescue in $m d x$ mice relies on upregulation of utrophin, the effects of SSPN overexpression on cardiac function were determined in both $m d x$ and $m d x$ :utr-het mice. We first evaluated left ventricular mass (LVmass) and heart weight normalized to tibia length (HW/TL). At 11-12 months of age, $m d x^{\mathrm{TG}}$ mice exhibited a nonsignificant reduction in HW/TL compared with $m d x$ controls (Figure 2A and Table 1). HW/TL values were only slightly improved in $m d x$ :utr-het mice compared with $m d \mathrm{x}$ :utr-het ${ }^{\mathrm{TG}}$ mice. Echocardiography measurements of LVmass indicated a significant reduction in ventricular mass of $m d x^{\mathrm{TG}}$ hearts relative to controls, whereas ventricular mass was similar in $m d \mathrm{x}$ :utr-het hearts compared with controls (Figure 2B and Table 1). Echocardiographic measurements of ventricular dimensions revealed that left ventricular end-diastolic (LVEDD) and end-systolic dimension (LVESD) were affected by SSPN-Tg expression, which indicated a reduction in pathological ventricular remodeling upon SSPN overexpression (Figure 2, C and D, and Table 1). LVEDD values trended toward smaller values in $m d x^{\mathrm{TG}}$ mice compared with $m d x$ mice, whereas LVESD were significantly smaller in $m d x$ hearts compared with $m d x^{\mathrm{TG}}$ hearts. In $m d x$ :utr-het mice, LVEDD and LVESD values were significantly greater compared with those of $m d x$ controls (Figure $2 \mathrm{C}$ and Table 1). A reduction in DMD-associated ventricular remodeling was also evident in ventricular dimensions of SSPN-Tg mice, as both LVEDD and LVESD values in $m d x$ :utr-het ${ }^{\mathrm{TG}}$ hearts were significantly reduced compared with those in $m d x$ :utr-het hearts (Figure $2 \mathrm{D}$ and Table 1 ). Our analysis revealed that $m d x$ :utr-het mice displayed more advanced pathologic ventricular remodeling by a younger age ( $\sim 39$ weeks) compared with $m d x$ mice ( $\sim 50$ weeks). In both DMD mouse models, overexpression of SSPN improved ventricular geometries in both diastole and systole. 
A

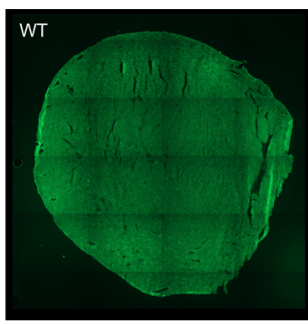

B

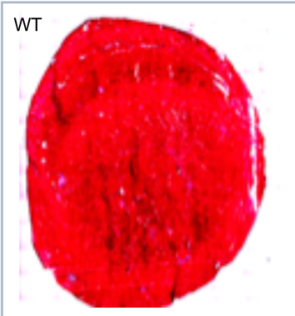

C

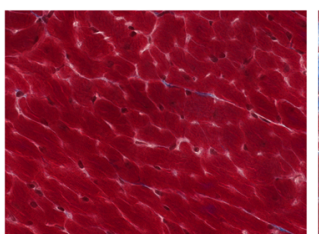

D

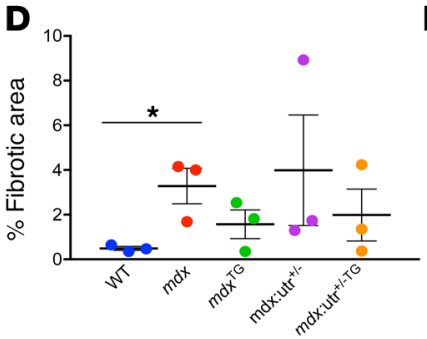

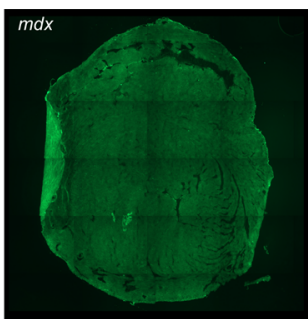

$m d x$
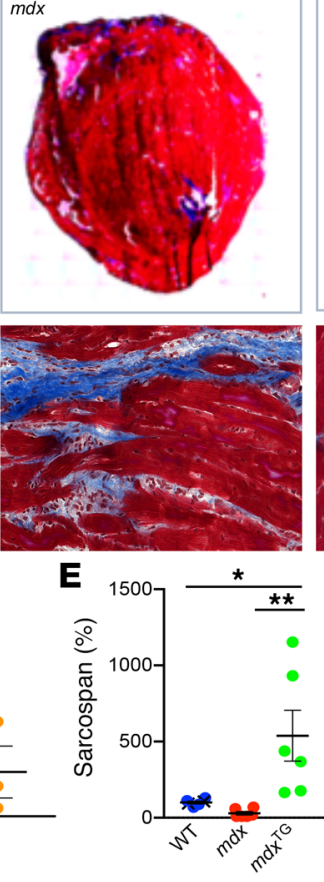
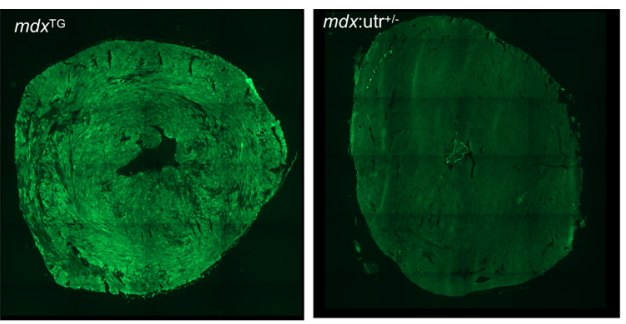

$m d x^{\top \mathrm{G}}$
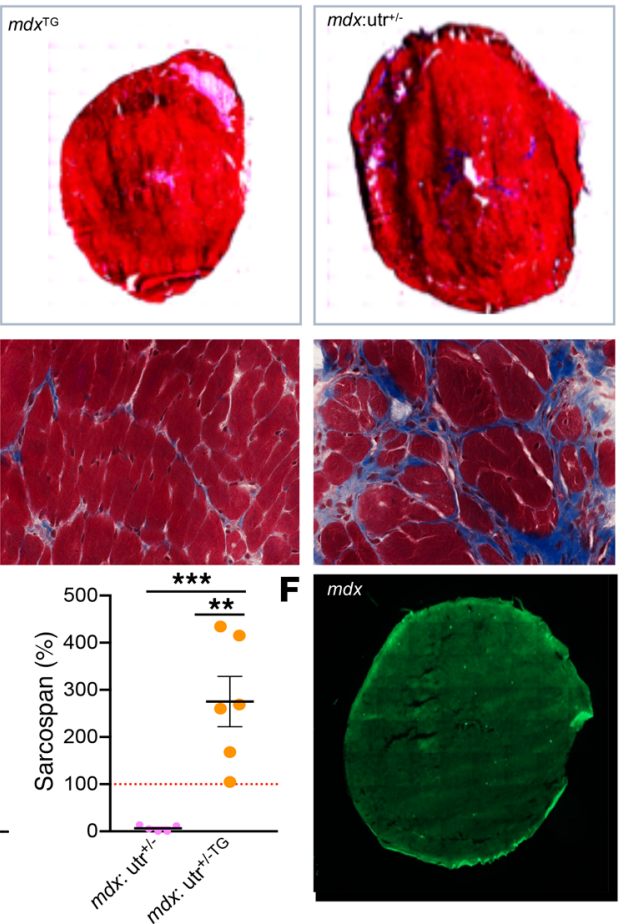
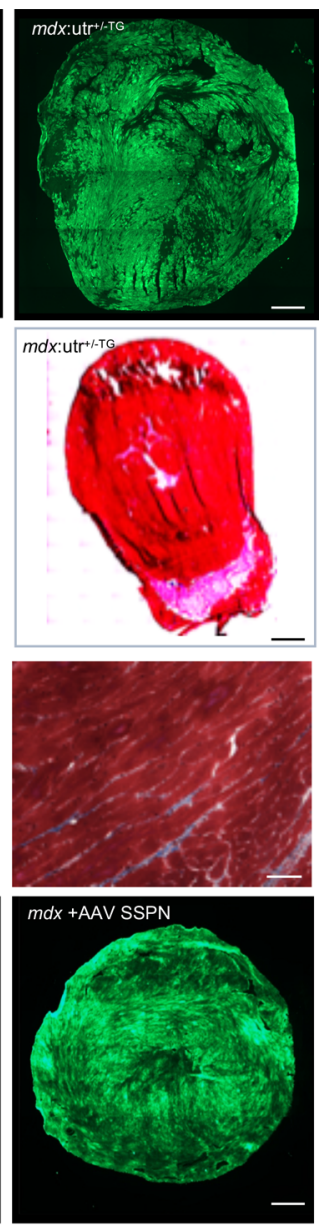

Figure 1. Sarcospan overexpression improves cardiac histology in DMD mouse models. (A) Indirect immunofluorescence detection of SSPN antibody staining of whole heart cryosections from WT, $m d x$, and $m d x$ :utr-het ( $\left.m d x: \mathrm{utr}^{+/-}\right)$mice and SSPN-overexpressing SSPN-Tg ( $m d x^{\mathrm{TC}}$ and $m d x$ :utr $\left.{ }^{+-\mathrm{Tg}}\right)$ mice. Staining represents both endogenous and Tg SSPN expression in hearts of indicated genotypes $(n=4)$. Scale bar: $900 \mu \mathrm{m}$. (B) Masson's trichrome (MT) staining reflects collagen abundance in transverse whole cardiac cross sections from indicated genotypes $(n=3)$. Original magnification, $\times 10$ (whole heart images). Scale bar: $900 \mu \mathrm{m}$. (C) MT-stained transverse cardiac cross sections ( $n=3$ biological replicates per genotype). Original magnification, $\times 20$. Scale bar: $50 \mu \mathrm{m}$. (D) Quantification of the percentage of fibrotic area in transverse cardiac cross sections determined by analysis of MT collagen staining relative to total area. Data presented are from individual animals, and data are presented as mean \pm SEM $(n=3)$. (E) Intensity of sarcolemma SSPN staining is quantified from immunofluorescence images ( $n=3$ biological replicates, $n=6$ technical replicates), and data are presented as mean \pm SEM. For all data shown statistics were determined by 1-way ANOVA followed by Tukey's multiple comparisons test. $P \leq 0.05$ was considered significant. ${ }^{*} P \leq$ $0.05,{ }^{* *} P \leq 0.01,{ }^{* *} P \leq 0.001$. (F) Transverse whole cardiac cryosections stained with SSPN antibodies reveal robust SSPN expression achieved in $m d x$ hearts upon systemic administration of AAV6 SSPN $(n=3)$. Scale bar: $900 \mu \mathrm{m}$.

The less severe $m d x$ model maintained a relatively preserved left ventricular function (left ventricular ejection fraction [LV EF\%] and left ventricular fractional shortening [LV FS\%]) with respective values of $58.9 \% \pm 1.61 \%$ and $31.0 \% \pm 1.17 \%$, indicating preservation of ventricular contractile performance at this disease stage. The $m d x^{\mathrm{TG}}$ mice had significantly higher LV EF\% and LV FS\% values compared with those of $m d x$ controls (Figure 2, E and F, and Table 1). In the more severe $m d x$ :utr-het mouse model, SSPN overexpression significantly improved LV EF\% and LV FS\% outcomes, such that mdx:utr-het ${ }^{\mathrm{TC}}$ mice exhibited higher values than non-Tg (NTG) controls (Figure 2, E and F). In this study, 10- to 11-month-old $m d x$ :utr-het mice had reduced contractile performance compared with 12-month-old $m d x$ mice (Table 1). Therefore, overexpression of SSPN in $m d x^{\mathrm{TG}}$ hearts significantly improved left ventricular contractile function, as reflected by a comparison of $m d x^{\mathrm{TG}} \mathrm{LV} \mathrm{EF} \%$ and $\mathrm{LV} \mathrm{FS \%} \mathrm{values} \mathrm{relative} \mathrm{to}$ those of $m d x$ controls. A summary of the baseline echocardiography data are provided (Table 1).

Hemodynamic studies reveal that SSPN overexpression improves the contractile performance characteristic of $D M D$ cardiomyopathy. To determine effects of SSPN overexpression on cardiac function, we evaluated $m d x$ mice using catheter-based hemodynamic studies. We utilized pressure-volume analysis to investigate SSPN 
A
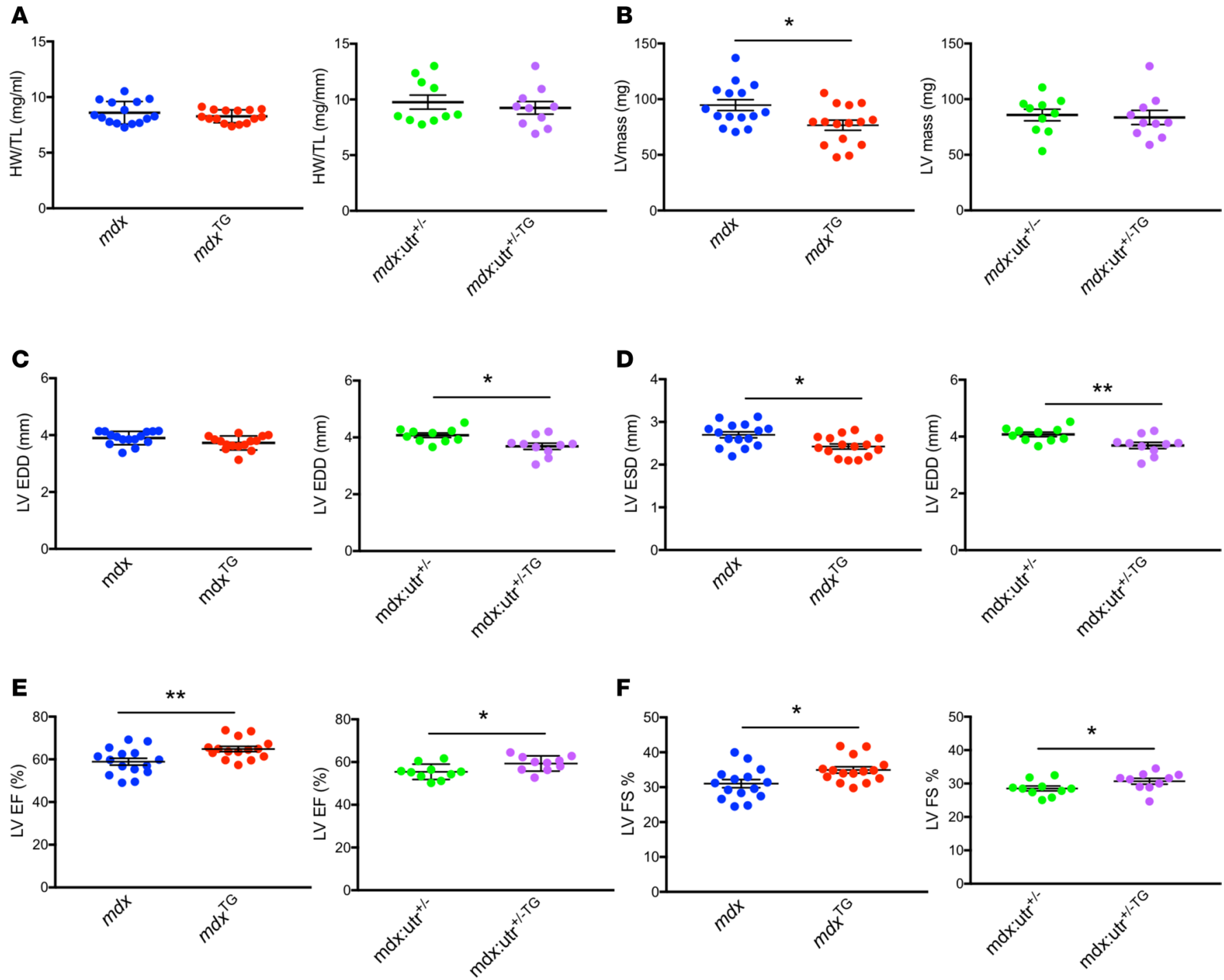

Figure 2. Sarcospan overexpression improves baseline cardiac parameters in DMD mouse models. Echocardiographic measurements were obtained for $m d x(n=15), m d x^{\top \mathrm{TC}}(n=15), m d x$ :utr-het $\left(m d x: \mathrm{utr}^{+/-}\right)(n=10)$, and $m d x$ :utr-het ${ }^{\mathrm{TC}}\left(m d x\right.$ :utr $\left.{ }^{+/ \mathrm{TC}}\right)(n=10)$ mice are shown in A-C. (A) Heart weight/tibia length (HW/TL) and echocardiography results. (B) LVmass measurements indicate degree of cardiac hypertrophy. (C) Left ventricular end-diastolic dimension (LVEDD) and (D) left ventricular end-systolic dimension (LVESD). (E) Values obtained for left ventricular ejection fraction (LV EF\%) and (F) left ventricular fractional shortening (LV FS\%). Individual data points are plotted for each animal, and data are presented as mean \pm SEM. Statistics were determined by Student's $t$ test between disease models ( $m d x$ vs. $m d x^{\top \mathrm{C}}$ and $m d x$ :utr-het vs. $m d x$ :utr-het ${ }^{\top \mathrm{C}}$ ). $P \leq 0.05$ was considered significant. ${ }^{*} P \leq 0.05 ;{ }^{* *} P \leq 0.01$. A summary of all data and values is provided in Table 1.

overexpression on the $m d x$ background, since $m d x$ mice demonstrate profiles characteristic of dystrophic cardiac disease (Figure 3A). Therefore, this approach allowed us to better determine the physiological parameters affected by SSPN overexpression. The $m d x$ mice evaluated in this study were approximately 11 months of age. Catheter-based measurements examining global contractility of the left ventricle revealed changes in ventricular pressures during the systolic interval ( $\mathrm{dP} / \mathrm{dt}_{\max }$ ). The systolic pressures were significantly reduced in $m d x$ mice, reflecting diminished contractile and possibly vascular performance. SSPNTg overexpression in $m d x$ mice normalized systolic performance, as indicated by $\mathrm{dP} / \mathrm{dt}_{\max }$ measurements (Figure 3B and Table 2). As illustrated by the 2 representative PV loops (Figure 3A), the $m d x^{\mathrm{TG}} \mathrm{PV}$ loop displayed a calculated slope of the end-systolic pressure-volume relationship (ESPVR) was greater in $m d x^{\mathrm{TG}}$ and PRSW values that were higher than that calculated from the $m d x$ PV loop. Decreased ESPVR slope and PRSW values indicated a reduction in contractility and systolic function. In this study, the average ESPVR slope values for $m d x^{T G}$ mice were $16.3 \%$ higher than in $m d x$ mice (Figure $3 \mathrm{~A}$ ). Analysis of end-systolic pressure also revealed improvements in systolic function in $m d x^{\mathrm{TG}}$ hearts (Figure $3 \mathrm{~B}$ and Table 2). 
Table 1. Summary of baseline echocardiographic data from $m d x$ and $m d x$ :utr-het mice reveals that SSPN improves contractile function

\begin{tabular}{|c|c|c|c|c|}
\hline LVEDD (mm) & $3.90 \pm 0.06$ & $3.73 \pm 0.06$ & $4.08 \pm 0.25$ & $3.69 \pm 0.11^{\mathrm{B}}$ \\
\hline LV EF (\%) & $58.9 \pm 1.61$ & $64.9 \pm 1.23^{A}$ & $55.4 \pm 1.14$ & $59.3 \pm 1.12^{\mathrm{B}}$ \\
\hline LV FS (\%) & $31.0 \pm 1.17$ & $34.9 \pm 0.93^{A}$ & $28.5 \pm 0.73$ & $30.7 \pm 0.87^{B}$ \\
\hline $\mathrm{SV}(\mathrm{ml})$ & $38.8 \pm 1.39$ & $38.5 \pm 1.49$ & $40.6 \pm 1.59$ & $34.6 \pm 2.28^{B}$ \\
\hline MV E/A & $1.28 \pm 0.06$ & $1.45 \pm 0.09$ & $1.40 \pm 0.11$ & $1.44 \pm 0.07$ \\
\hline PWT;D (mm) & $0.79 \pm 0.04$ & $0.73 \pm 0.04$ & $0.66 \pm 0.08$ & $0.78 \pm 0.05$ \\
\hline PWT;S (mm) & $1.09 \pm 0.04$ & $1.12 \pm 0.03$ & $1.01 \pm 0.07$ & $1.08 \pm 0.04$ \\
\hline IVS (mm) & $0.94 \pm 0.04$ & $0.80 \pm 0.05^{A}$ & $0.87 \pm 0.06$ & $0.90 \pm 0.06$ \\
\hline LVmass (mg) & $94.7 \pm 4.88$ & $76.6 \pm 4.56^{A}$ & $85.8 \pm 4.80$ & $83.6 \pm 6.37$ \\
\hline
\end{tabular}

Values are reported as averages (mean $\pm \mathrm{SEM}$ ). Echocardiography was performed on $m d x(\mathrm{DMD}) ; m d x^{\mathrm{TC}} ; m d x$ : utr-het; and $m d x$ :utr-het ${ }^{\mathrm{TC}}$ mice.

Measurements reported are heart rate (HR); left ventricular (LV) end-diastolic dimension (LVEDD); LV end-systolic dimension (LVESD); LV EF (\%), LV ejection fraction percentage; LV FS (\%), LV fractional shortening percentage; stroke volume (SV); mitral valve (MV); early peak flow velocity (E); atrial peak flow velocity (A); mitral valve early peak flow velocity/atrial peak flow velocity (MV E/A); diastolic posterior wall thickness (PWT;D); systolic posterior wall thickness (PWT;S); interventricular septum thickness (IVS); LV mass; and heart weight/tibia length (HW/TL). Individual Student's $t$ test was used to compare differences between paired groups: $m d x$ and $m d x^{\top \mathrm{TC}}(12 \mathrm{mo})$ and $m d x$ :utr-het and $m d x$ :utr-het ${ }^{\top \mathrm{CC}}(10 \mathrm{mo})$. ${ }^{\mathrm{A}} P<0.05$ (comparison between $m d x$ and $m d x^{T C}$ mice); ${ }^{\mathrm{B}} P<0.05$ (comparison between $m d x$ :utr-het and $m d x$ :utr-het ${ }^{\mathrm{TC}}$ mice).

We also assessed whether SSPN overexpression improved diastolic relaxation (lusitropy) in $m d x$ hearts, since diastolic dysfunction developed during early stages of disease. Histological evaluation of the $m d x$ mice revealed marked levels of fibrosis (Figure 1B), which may affect cardiac function. Measurements of diastolic left ventricular pressures of $m d x$ and $m d x^{\mathrm{TG}}$ mice indicated that the $\mathrm{Tg} m d x$ mice showed no significant changes in $\mathrm{dP} / \mathrm{dt}_{\min }$ values relative to those of $m d x$ controls (Figure $3 \mathrm{C}$ ). Individual assessments of end-diastolic pressures (Ped) and end-diastolic volumes (Ved) in $m d x$ mice indicated that pressures were reduced with larger volumes, likely reflecting greater ventricular chamber sizes. The value for Ped in $m d x^{\mathrm{TG}}$ mice (Figure 3C and Table 2) was improved (higher) compared with $m d x$ mice. Furthermore, there was no difference detected in Ved between $m d x$ and $m d x^{\mathrm{TG}}$ mice. In Figure 3D, values reported for pressure measurements (pressure at maximum rate of change in pressure over time $\left[\mathrm{P} @ \mathrm{dP} / \mathrm{dt}_{\max }\right]$ ) and mean ventricular pressure were significantly increased in $m d x^{\mathrm{TG}}$ compared with NTG $m d x$ controls (Figure 3D and Table 2). Whereas, values for P@ dV/dt ${ }_{\max }$ were not significantly different (Figure 3D and Table 2).

Additional parameters measured during pressure-volume studies provide a more refined view of the effects of SSPN overexpression on $m d x$ cardiac function. Stroke work (SW) in $m d x^{\mathrm{TG}}$ hearts was significantly higher than $m d x$ controls (Figure $3 \mathrm{E}$ and Table 2). Increased SW reflected the area enclosed by the PV loop and depended on the stroke volume (SV) and afterload (mean aortic/pulmonary arterial pressure). The significant increase in SW values in $m d x^{\mathrm{TG}}$ mice may be due to increased afterload by effects on the vasculature, especially since SV was largely similar between $m d x$ and $m d x^{\mathrm{TG}}$ mice, (Figure $3 \mathrm{E}$ and Table 2). Cardiac output (CO) is dependent on SV and heart rate and was similar between the genotypes analyzed. As expected, the $m d x$ and $m d x^{\mathrm{TG}}$ mice exhibited similar $\mathrm{CO}$ values (Figure $3 \mathrm{E}$ and Table 2).

SSPN improvement of cardiac performance in DMD mice is attributed to utrophin stabilization at the sarcolemma. Immunofluorescence imaging was utilized to assess effects of Tg overexpression of SSPN in $m d x$ mice to assess dependence of SSPN rescue on upregulation of utrophin as well as other proteins important in cell membrane stabilization. Transverse cardiac cryosections were stained with antibodies against components of laminin-binding adhesion complexes, including the DGC. As expected, dystrophin seen in WT hearts, was not restored by SSPN overexpression in $m d x$ hearts (Figure 4A). We focused our investigation on the UGC and the $\alpha 7 \beta 1$ integrin complex, as they are known to compensate for loss of dystrophin and, when overexpressed, can ameliorate muscular dystrophy by restoring muscle cell adhesion. Hearts were stained 
A
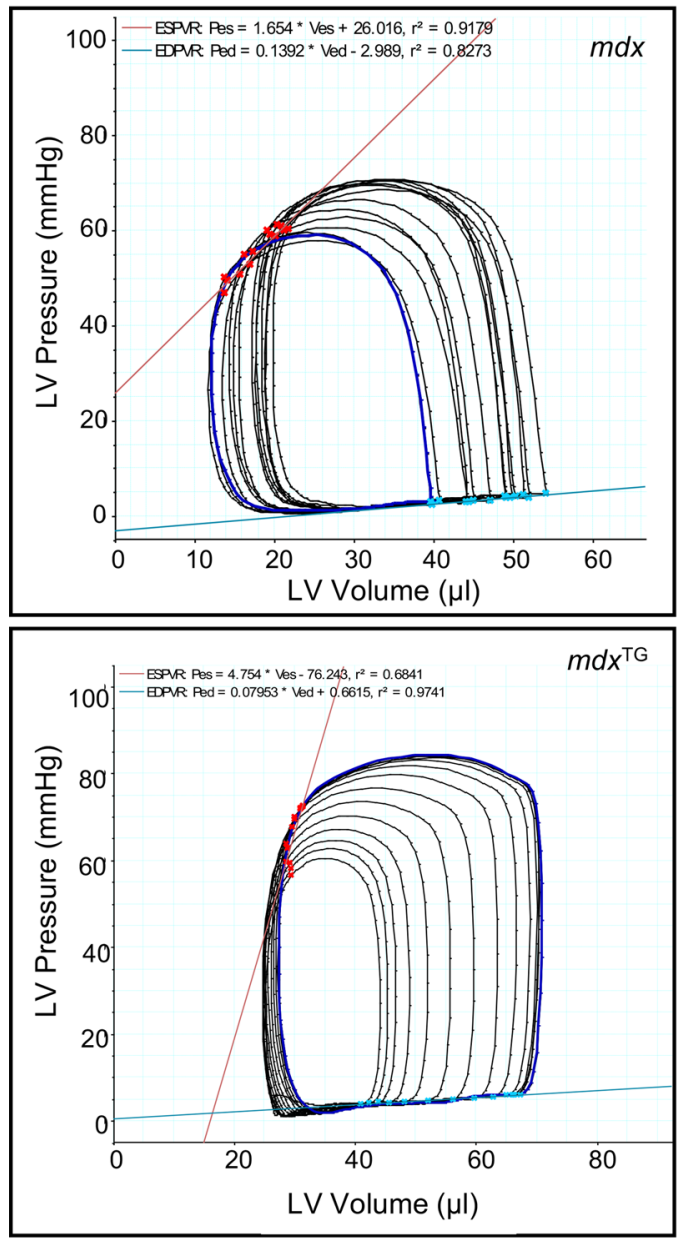

B Systolic Function
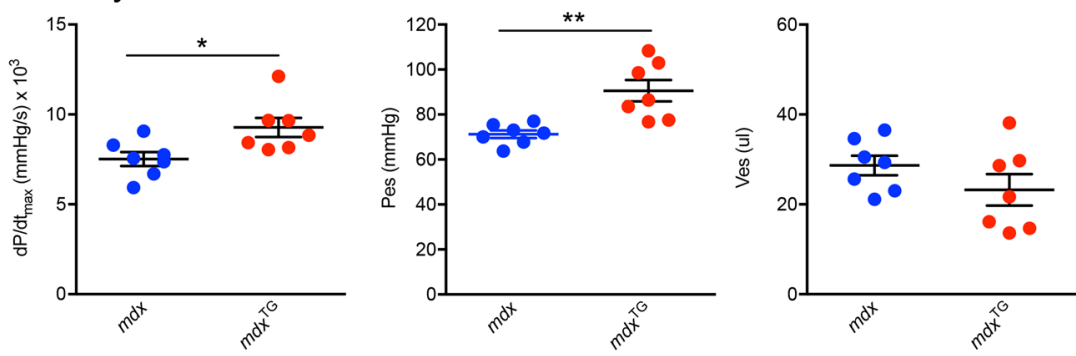

\section{Diastolic Function}
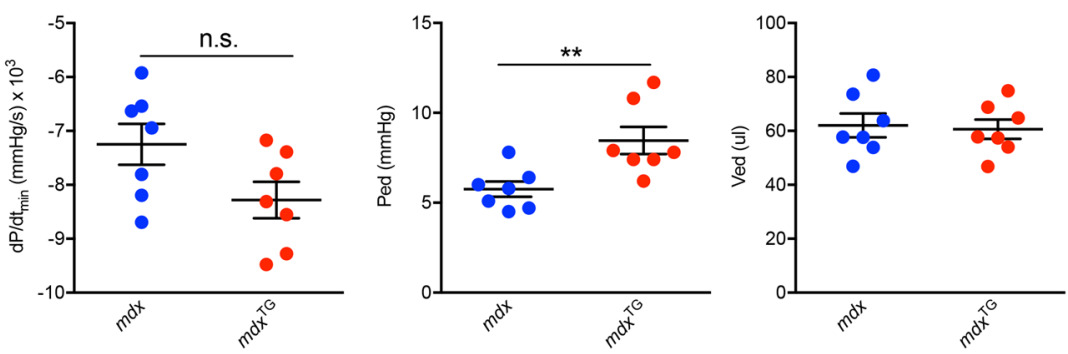

D Pressure Measurements
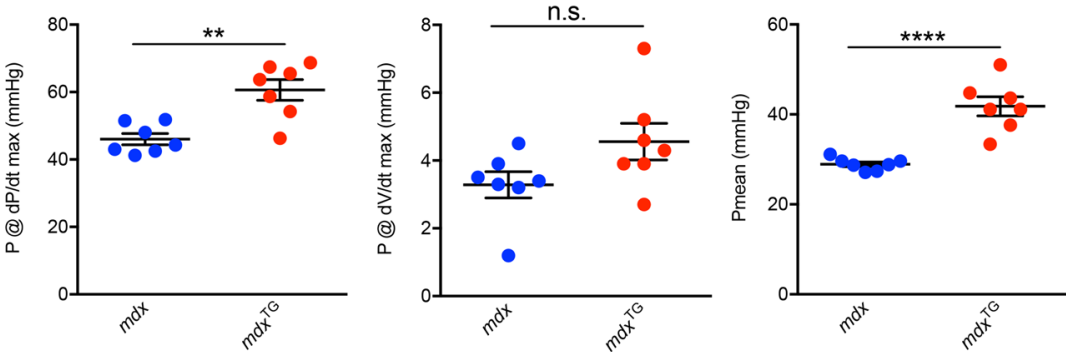

E Output Measurements
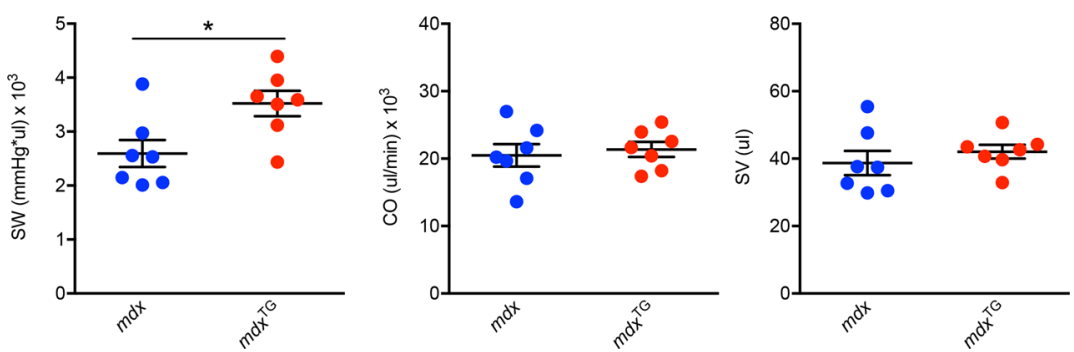

Figure 3. Hemodynamic studies reveal that sarcospan improves contractile performance. Pressure-volume measurements were conducted in $m d x$ ( $n$ $=7$ ) and $m d x^{T C}$ mice $(n=7)$. (A) Representative pressure-volume loops are shown for $m d x$ and $m d x^{\top \mathrm{TC}}$ mice. (B) Systolic function measurements include $\mathrm{dp} / \mathrm{dt}_{\text {max }^{\prime}}$ end-systolic pressure (Pes), and end-systolic volume (Ves). (C) Measurements reporting diastolic function include $\mathrm{dp}_{\mathrm{dt}} \mathrm{min}$, end-diastolic

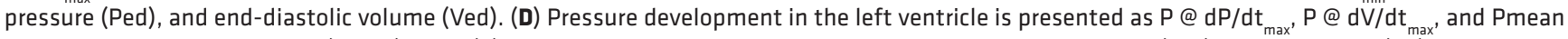
for the $m d x$ and SSPN-Tg $m d x\left(m d x^{T C}\right)$ mice. (E) Evaluation of the heart's work capacity, including stroke work (SW), cardiac output (CO), and stroke volume (SV) parameters. Individual data points are plotted from each animal, and data are presented as mean \pm SEM. Statistics were determined by Student's $t$ test between relevant groups, and $P \leq 0.05$ was considered significant. ${ }^{*} P \leq 0.05,{ }^{* *} P \leq 0.01,{ }^{* * *} P \leq 0.0001$. A summary of all data and values is provided in Table 2 .

with antibodies against utrophin and $\beta 1 \mathrm{D}$ integrin, markers of these 2 adhesion complexes. In $m d x$ hearts, utrophin expression appeared bimodally affected; some hearts had substantial upregulation of utrophin and there was no noticeable upregulation in other hearts. This may be biological variability or it may be reflective of variations in disease progression within the cohort; it suggests that utrophin levels may be affected as part of disease pathogenesis. On average, $m d x^{\mathrm{TG}}$ hearts showed no significant effect on utrophin upregulation; however, there was improved consistency of utrophin at the sarcolemma in all $m d x^{\mathrm{TG}}$ hearts (Figure $4 \mathrm{~A}$ ). In WT hearts, utrophin staining at the sarcolemma was typically discontinuous. In contrast, dystrophin-defi- 
Table 2. Summary of pressure-volume studies in $\mathrm{mdx}$ mice reveal that SSPN prevents cardiac decline

\begin{tabular}{|c|c|c|}
\hline ECHO parameter & $m d x(n=7)$ & $m d x^{\mathrm{TC}}(n=7)$ \\
\hline HR (bpm) & $532.7 \pm 19.4$ & $509.2 \pm 16.6$ \\
\hline VeS $(\mu l)$ & $28.7 \pm 2.2$ & $23.2 \pm 3.5$ \\
\hline $\operatorname{VeD}(\mu \mathrm{l})$ & $62.0 \pm 4.4$ & $60.6 \pm 3.6$ \\
\hline $\mathrm{SV}(\mu \mathrm{l})$ & $38.7 \pm 3.6$ & $42.0 \pm 2.0$ \\
\hline $\mathrm{CO}(\mu \mathrm{l} / \mathrm{min})$ & $20473 \pm 1664$ & $21366 \pm 1103$ \\
\hline $\operatorname{LV} E F(\%)$ & $62.6 \pm 1.86$ & $69.6 \pm 3.63$ \\
\hline Ped (mmHg) & $5.76 \pm 0.4$ & $8.46 \pm 0.8^{A}$ \\
\hline Pes (mmHg) & $71.2 \pm 1.71$ & $90.6 \pm 4.77^{A}$ \\
\hline Tau (ms) & $5.90 \pm 0.24$ & $6.59 \pm 0.29$ \\
\hline $\mathrm{dP} / \mathrm{dt}_{\max }(\mathrm{mmHg} / \mathrm{s})$ & $7524 \pm 386$ & $9276 \pm 535^{A}$ \\
\hline $\mathrm{dP} / \mathrm{dt}_{\min }(\mathrm{mmHg} / \mathrm{s})$ & $-7247 \pm 379$ & $-8282 \pm 337$ \\
\hline $\mathrm{Ea}(\mathrm{mm} \mathrm{Hg} \mathrm{ml-1)}$ & $1.94 \pm 0.18$ & $2.20 \pm 0.14$ \\
\hline $\mathrm{SW}(\mathrm{mmHg} \times \mu \mathrm{l})$ & $2594 \pm 251$ & $3521 \pm 235^{A}$ \\
\hline EDPVR & $0.08 \pm 0.02$ & $0.07 \pm 0.01$ \\
\hline ESPVR & $1.95 \pm 0.51$ & $2.33 \pm 0.64$ \\
\hline PRSW & $66.0 \pm 5.33$ & $64.9 \pm 5.83$ \\
\hline PMean & $28.9 \pm 0.52$ & $41.8 \pm 2.11^{A}$ \\
\hline PMax & $79.5 \pm 1.17$ & $97.6 \pm 4.38^{A}$ \\
\hline \multicolumn{3}{|c|}{ 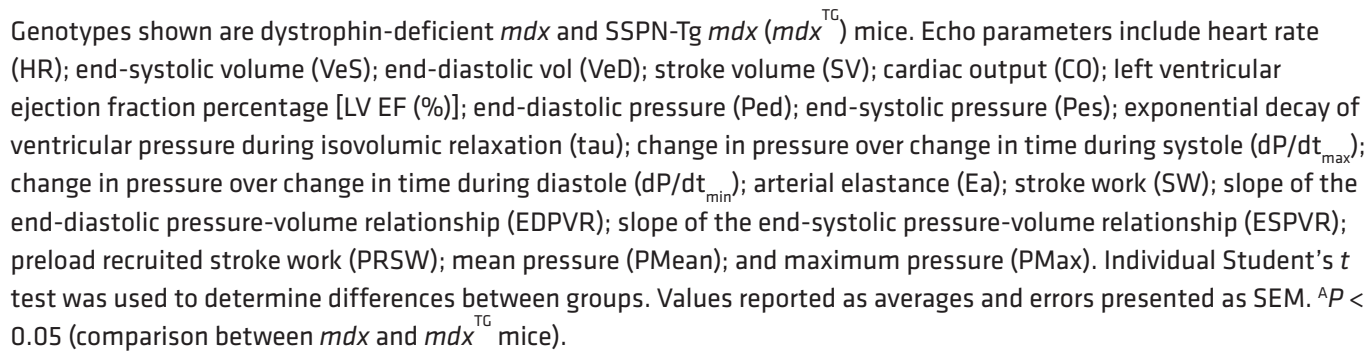 } \\
\hline
\end{tabular}

cient $m d x$ hearts assumed a more sarcolemmal distribution of utrophin that was more homogeneous than in $m d x^{\mathrm{TG}}$ hearts, which likely reflects the utrophin compensatory response (Figure 4, A and B). Itgb expression appeared similar in $m d x$ hearts compared with WT hearts, while $m d x^{\mathrm{TG}}$ hearts exhibited a further increase in Itgb at the sarcolemma compared with WT and $m d x$ hearts (Figure $4, \mathrm{~A}$ and C).

Another factor that influences membrane stability is $\alpha$-DG glycosylation $(35,36)$. Although in contrast to those in skeletal muscle, DGC proteins are largely retained at the cardiac sarcolemma in dystrophin deficiency $(21,37)$. Using indirect immunofluorescence of cardiac cryosections, we showed that glycosylated $\alpha$-DG (detected using anti-carbohydrate-specific antibody, IIH6) was retained at the sarcolemma of $m d x$ hearts, although levels were reduced compared with WT hearts (Figure 4, A and C). Overexpression of SSPN partially restored the expression of glycosylated $\alpha-\mathrm{DG}$ in $m d x^{\mathrm{TG}}$ hearts (Figure $4, \mathrm{~B}$ and C). To determine whether the reduction in IIH6 staining was due to loss of the protein, an antibody generated against the core DG protein (45-3) was used to detect the DG protein, independent of glycosylation status. Sarcolemmal staining of core DG protein appeared largely similar among WT, $m d x$, and $m d x^{\mathrm{TG}}$ hearts (Figure $4 \mathrm{~A}$ ). This indicates that differences in IIH6 staining may be primarily due to altered glycosylation of $\alpha$-DG. Examination of $\beta$-DG derived from the same gene product and propeptide showed similar protein levels as the core-DG and subtly increased levels in $m d x^{\mathrm{TG}}$ hearts (Figure $4 \mathrm{~A}$ ). The sarcoglycans ( $\alpha$-SG, $\beta$-SG, and $\gamma$-SG) were reduced in $m d x$ hearts compared with WT hearts (Figure 4A). SSPN overexpression in $m d x$ hearts enhanced abundance of these 3 sarcoglycans at the sarcolemma and significantly increased $\alpha$-SG (Figure 4C).

To determine whether the SSPN-Tg enhanced sarcolemmal expression of utrophin in $m d x$ :utr-het hearts, immunofluorescence images were examined. Utrophin levels trended toward modestly increased (Figure 5, A-C). Again, dystrophin was not restored by SSPN overexpression in $m d x$ :utr-het hearts (Figure $5 \mathrm{~A}$ ). Furthermore, utrophin and Itgb expression levels were lower in $m d x$ :utr-het hearts compared with $m d x$ hearts (Figure $4 \mathrm{C}$ and Figure $5 \mathrm{C}$ ). Only sarcolemma levels of Itgb were enhanced by SSPN-Tg overexpression in $m d x$ :utr-het hearts (Figure 5, A and C). It appeared that reduced utrophin expression 
A
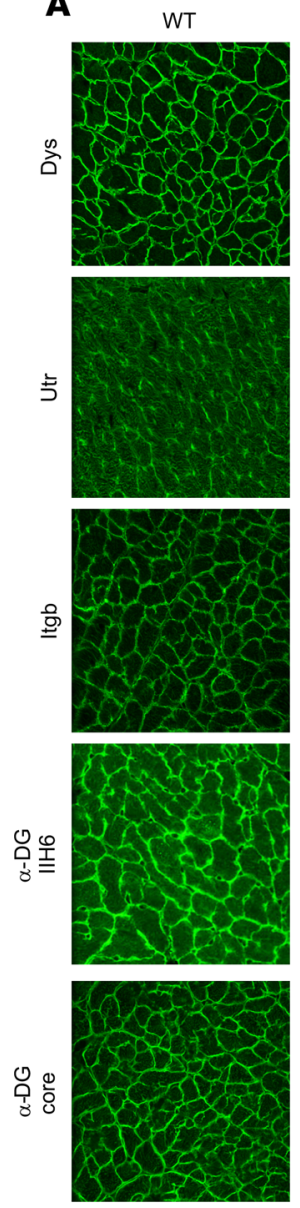

$m d x$
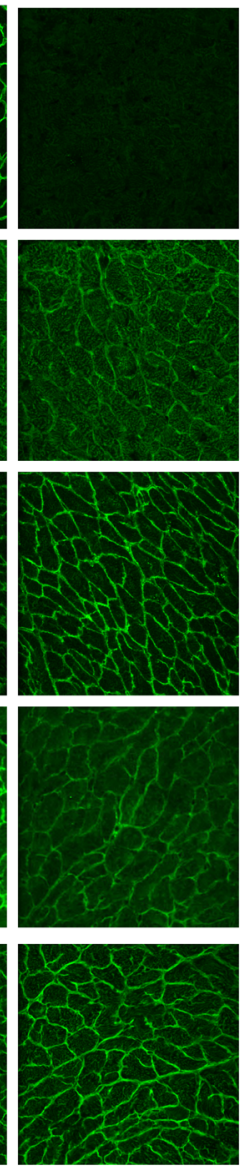

$m d x^{\top G}$
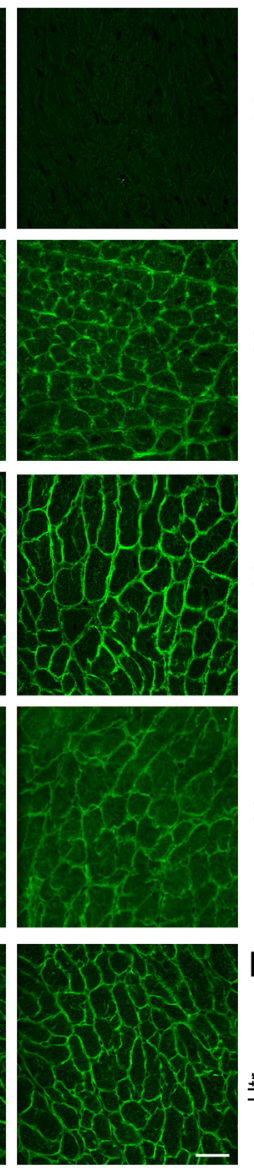
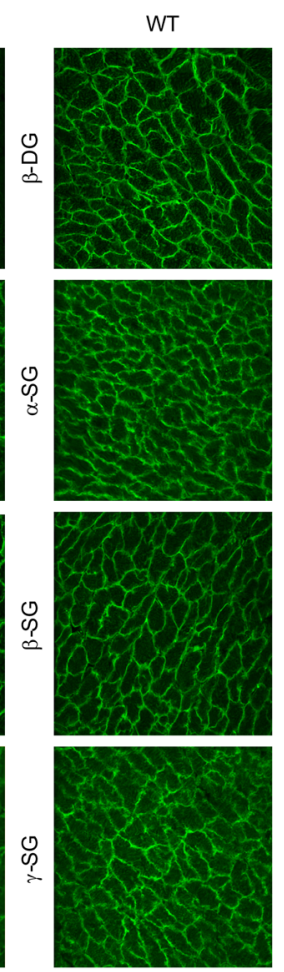

B

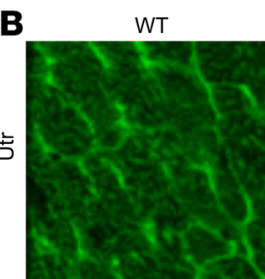

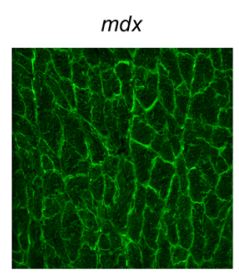
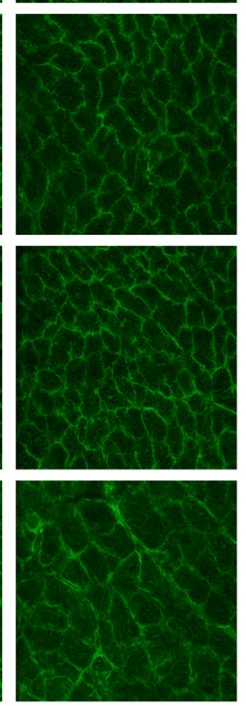

$m d x$

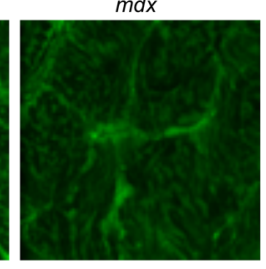

$m d x^{\top \mathrm{T}}$
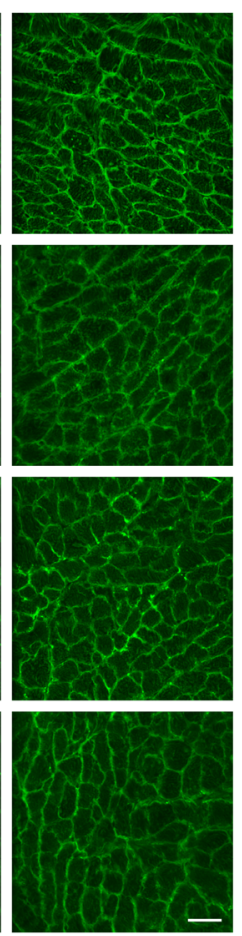

$m d x^{\top \mathrm{G}}$

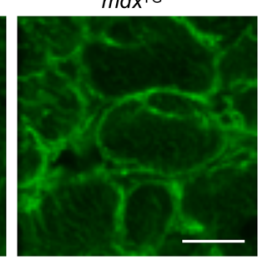

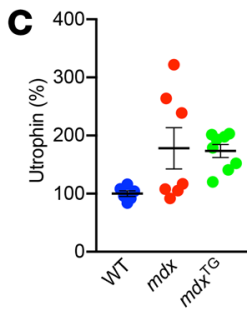
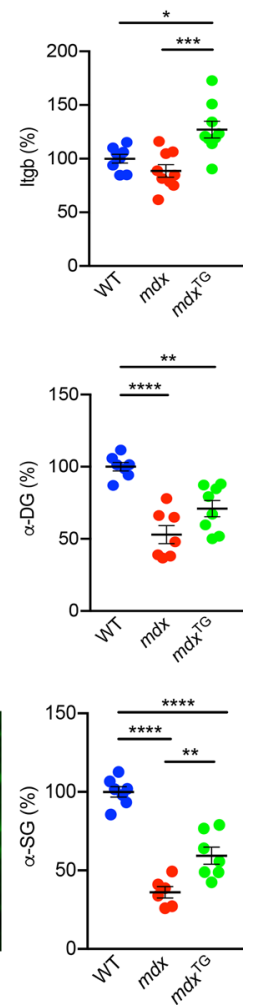

Figure 4. SSPN improvement of cardiac performance in DMD mice cannot be attributed to utrophin upregulation. (A) Confocal immunofluorescence images of transverse cross sections of $\mathrm{WT}, m d x, m d x^{\top \mathrm{TC}}$ hearts (original magnification, $\times 20$ ). To assess levels of major structural proteins associated with DMD, antibodies were utilized for dystrophin (Dys), utrophin (Utr), and Itgb ( $\beta 1 D$ integrin). Confocal settings were as follows: gain, 746.0 m/s, offset $-0.30 \%$; gain $788.6 \mathrm{~m} / \mathrm{s}$, offset $-0.30 \%$; gain $779.3 \mathrm{~m} / \mathrm{s}$, offset $-0.50 \%$, respectively. Expression of key DGC proteins important in stabilization and connection with laminin in the extracellular matrix (ECM) was assessed for $\alpha$-dystroglycan ( $\alpha$-DG), core-DG (45-3), and $\beta$-dystroglycan ( $\beta$-DC). Confocal settings were as follows: gain $733.6 \mathrm{~m} / \mathrm{s}$, offset $-0.10 \%$; gain: $819.5 \mathrm{~m} / \mathrm{s}$, offset $-0.30 \%$; gain $787.7 \mathrm{~m} / \mathrm{s}$, offset $-0.40 \%$, respectively. Sarcoglycans were upregulated at the DMD sarcolemma in hearts overexpressing SSPN. Antibodies utilized were specific for $\alpha$-sarcoglycan ( $\alpha$-SG), $\beta$-sarcoglycan ( $\beta$-SG), and $\gamma$-sarcoglycan $(\gamma$-SG). Scale bar: $50 \mu \mathrm{m}$. Confocal setting information is as follows: $\alpha$-SG - gain $630.1 \mathrm{~m} / \mathrm{s}$, offset $-0.50 \%$; $\beta$-SG - gain $719.5 \mathrm{~m} / \mathrm{s}$, offset $-1.80 \% ; \gamma$-SG - gain $721.3 \mathrm{~m} / \mathrm{s},-0.40 \%$ ( $n=3$ for each antibody and genotype). (B) Confocal immunofluorescence images of utrophin staining, Original magnification, $\times 63$. Confocal settings were as follows: gain $788.6 \mathrm{~m} / \mathrm{s}$, offset $-0.10 \%$. Scale bar: $100 \mu \mathrm{m}$. (C) Intensity of sarcolemma protein staining of utrophin, Itgb, $\alpha$-DG (IIH6), and $\alpha$-SG is quantified from immunofluorescence images and all groups were compared by 1-way ANOVA using Tukey's multiple comparison test ( $n=3$ biological replicates, $n=6$ analyzed images). Data are presented as mean \pm SEM. $P \leq 0.05$ was considered significant. ${ }^{*} P \leq 0.05,{ }^{* *} P \leq 0.01,{ }^{* *} P \leq 0.001,{ }^{* * *} P \leq 0.0001$.

in dystrophin-deficient $m d x$ :utr-het hearts further decreased the abundance of glycosylated $\alpha$-DG at the sarcolemma compared with $m d x$ hearts (Figure $4 \mathrm{C}$ and Figure $5 \mathrm{C}$ ). SSPN overexpression did not significantly enhance $\alpha-D G$ glycosylation or alter protein abundance of core-DG or $\beta$-DG in $m d x$ :utr-het ${ }^{\mathrm{TG}}$ hearts (Figure 5A). The sarcoglycans ( $\alpha$-SG, $\beta$-SG, and $\gamma$-SG) were further reduced in $m d x$ :utr-het hearts compared with WT hearts (Figure $5 \mathrm{C}$ ). In $m d x$ :utr-het hearts, the SSPN-Tg did not greatly increase expression of $\alpha$-SG, $\beta$-SG, and $\gamma$-SG at the sarcolemma (Figure 5, A and C).

SSPN protects dystrophic hearts from $\beta$-adrenergic-mediated membrane damage. In this study, we determined whether SSPN overexpression restored cardiovascular responsiveness to sympathetic activation through isoproterenol-mediated activation of $\beta$-adrenergic receptors. We were also interested in whether SSPN membrane stabilization protected the sarcolemma during conditions of increased cardiac demand. 
Isoproterenol administration to $m d x^{\mathrm{TG}}$ mice after PV loop analysis resulted in significantly increased heart rates (HRs) compared with those in the pretreatment group (Figure 6A). Similarly, isoproterenol treatment of $m d x^{\mathrm{TG}}$ mice significantly increased $\mathrm{dP} / \mathrm{dt}_{\max }(18.5 \%)$ compared with pretreatment values. However, $m d x$ mice did not exhibit significant increases in $\mathrm{dP} / \mathrm{dt}_{\max }$ and $\mathrm{HR}$ compared with pretreatment values (Figure $6 \mathrm{~A}$ and Table 3). Furthermore, $m d x^{\mathrm{TG}}$ mice exhibited greater baseline LV EF\% compared with $m d x$ mice. Isoproterenol dosing of $m d x$ and $m d x^{\mathrm{TG}}$ mice did not significantly increase $\mathrm{LV} \mathrm{EF} \%$, an expected response of WT mice. WT measurements indicated responsiveness to the isoproterenol-dosing regimen, and HR was slightly increased compared with values prior to treatment. Based on our results in WT mice, a higher isoproterenol dose appeared necessary to observe the documented significant changes in $\mathrm{HR}, \mathrm{dP} / \mathrm{dt} \max$, and $\mathrm{LV} \mathrm{EF} \%$. Ventricular pressures $\left(\mathrm{dP} / \mathrm{dt}_{\max }\right)$ typically rise in WT mice as a result of isoproterenol treatment; a nonsignificant increase $P=0.055$ was seen compared with pretreatment values; whereas pretreatment LV EF\% values were increased in WT mice after exposure to isoproterenol (Figure 6A and Table 3).

While WT hearts do not exhibit Evan's blue dye (EBD) uptake, membrane fragility defects are evident during aging and can be induced with cardiac stress. To determine whether SSPN overexpression improved membrane fragility of $m d x$ and $m d x$ :utr-het hearts, mice were subjected to an isoproterenol challenge protocol designed to increase work demand on the heart and to test the integrity of dystrophic cell membranes (Figure 6B). Mice were injected with the in vivo tracer EBD prior to $\beta$-adrenergic stimulation to track effects of increased work demand in SSPN-Tg and control dystrophic hearts. EBD images of whole transverse cardiac sections allowed visualization of the localization and the extent of EBD uptake in the untreated and isoproterenol-treated hearts (Figure 6C). Isoproterenol treatment of WT mice elicited low levels of EBD uptake (percentage EBD positive areas relative to total area), while $m d x$ mice exhibited a significant increase in EBD-positive areas, with multiple large and small areas of uptake (Figure 6C and Supplemental Figure 1B; supplemental material available online with this article; https://doi.org/10.1172/jci.insight.123855DS1). More EBD-positive areas were visible in areas of the heart adjacent to the lumen. The $m d x$ :utr-het hearts also displayed a similar large 80\% increase in EBD uptake compared with WT hearts, with a widespread distribution of EBD-positive areas similar to $m d x$ mouse hearts. In many regions, colocalization of SSPN staining with EBD-positive areas was reduced; therefore, regions with EBD uptake in Tg mice may have correlated with areas with lower levels of SSPN overexpression. The quantification of the percentage of EBD-positive areas was compared with the total area in 2 sections from each heart (mid and base) and is shown for mice of each genotype utilized in this study (Supplemental Figure 1B). Overall, SSPN-Tg mice after isoproterenol treatment had lower percentage EBD uptake compared with control, NTG mice of the same genotype (Figure 6C). The degree of cardiac damage (number of EBD-positive fibers and formation of large EBD-positive areas) in $m d x$ and $m d x$ :utr-het hearts provided insight into the effectiveness of SSPN in preventing formation of larger EBD-positive areas. Examining the total number of EBD-positive areas provided information about the number of areas with potential to undergo further damage when aggravated by stress (Supplemental Figure 1A). Overall, SSPN overexpression reduced the number of large EBD areas detected in both DMD mouse models (Supplemental Figure 1, A and B, and Table 4). SSPN overexpression significantly reduced the size of damaged areas and protected against further damage during induction of cardiac stress.

To assess whether increased abundance of sarcolemma adhesion complexes enhanced membrane integrity, we assessed membrane damage by examining the levels of cardiac creatine kinase MB isoform (CK-MB; ng/ml) in blood sera (Figure 6D). This assay indicated the degree of cardiac muscle membrane leakiness in untreated mouse models examined in this study (Figure 6D). WT mice had low levels of the CK-MB isoenzyme, whereas $m d x$ mice had a significant increase of CK-MB levels in their blood serum. The $m d x^{\mathrm{TG}}$ mice had significantly lower CK-MB values than $m d x$ mice, which were restored close to WT levels. Furthermore, $m d x$ :utr-het mice had similar serum CK-MB values as $m d x$ mice, which were substantially higher than WT mice. Tg SSPN overexpression significantly reduced CK-MB values of the more severe $m d x$ :utr-het disease model and restored blood serum levels close to levels in WT mice, demonstrating that SSPN protects the cardiac sarcolemma in the context of muscular dystrophy.

\section{Discussion}

Many promising therapeutic strategies exist on the horizon as potential means to end several of the debilitating aspects of the pediatric disease DMD. However, all therapeutic platforms face fundamental challenges or limitations that must be overcome to accomplish the intended goals. DMD remains $100 \%$ lethal in children and young adults, and the first accelerated approvals were granted by the FDA 
A
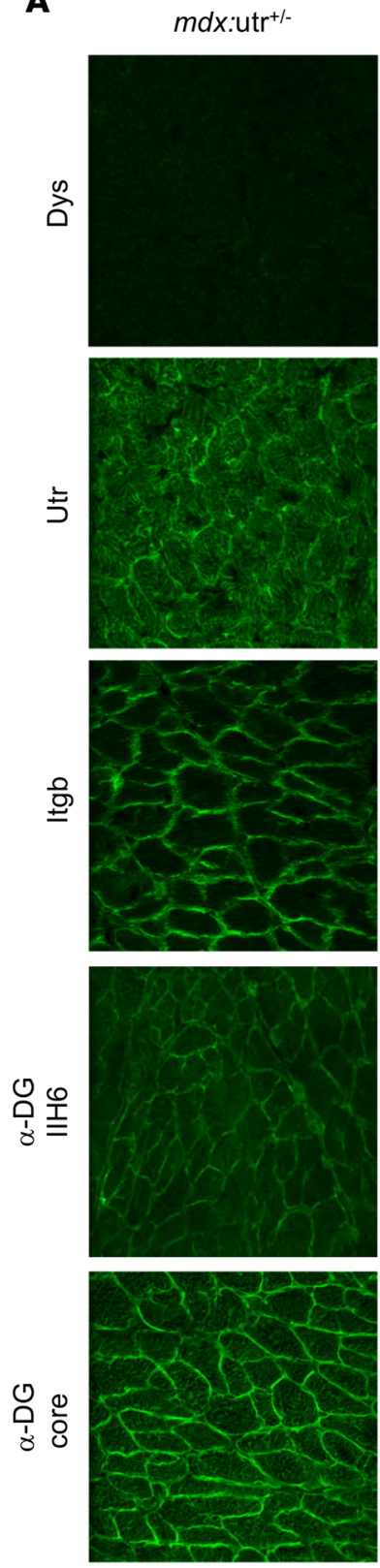

mdx:utr+/-TG
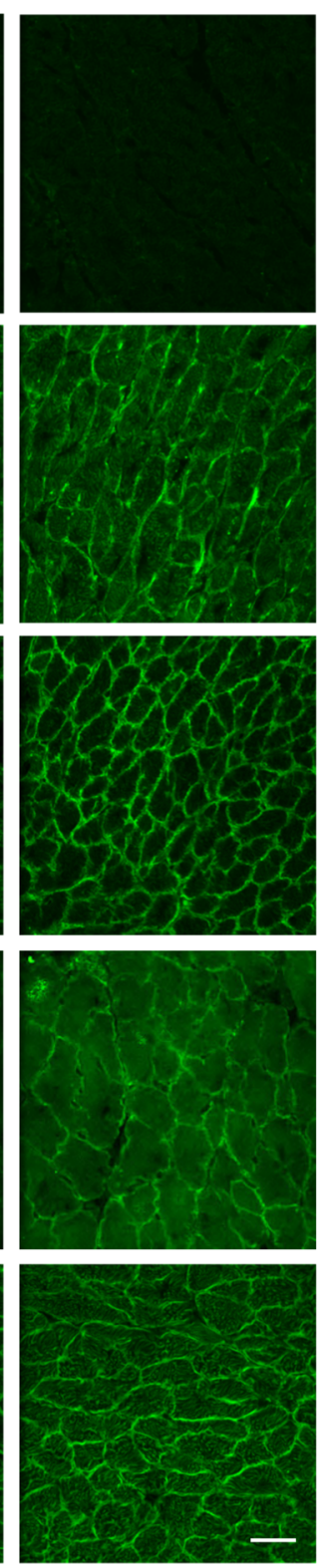
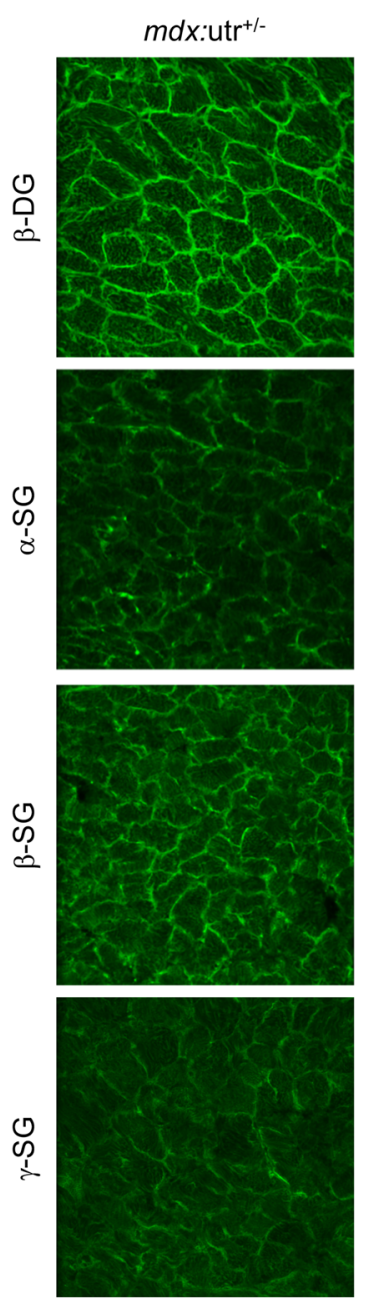

B

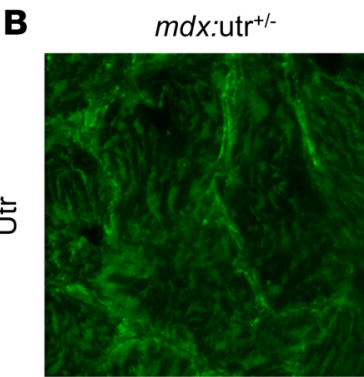

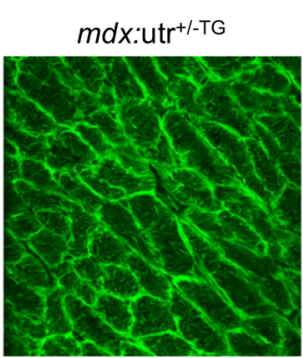
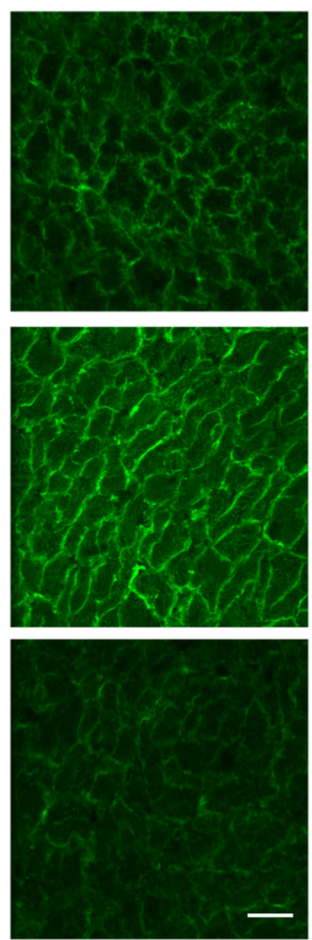

$m d x: u^{+l-T G}$

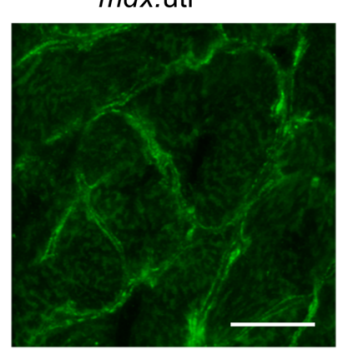

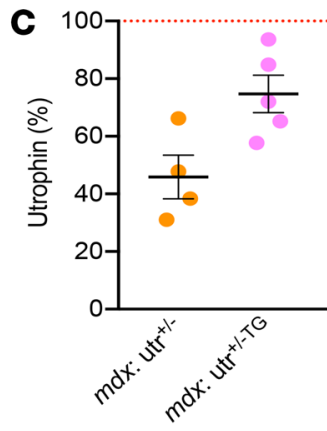
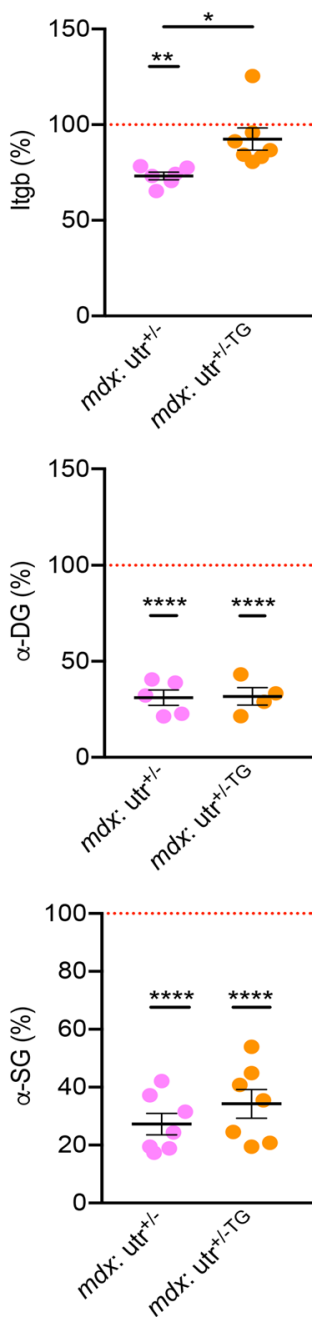

Figure 5. SSPN improvement of cardiac performance in DMD mice cannot be attributed to utrophin upregulation. (A) Confocal immunofluorescence images of transverse cross sections of $m d x$ :utr-het $\left(m d x: u t r^{+/}\right)$and $m d x$ :utr-het ${ }^{T C}\left(m d x: u_{t r}{ }^{+/-T g}\right)$ hearts. Original magnification, $\times 20$. To assess levels of major structural proteins associated with DMD, antibodies were utilized for dystrophin (Dys), utrophin (Utr), and Itgb ( $\beta 1 D$ integrin). Expression of key DGC proteins important in stabilization and connection with laminin in the extracellular matrix (ECM) were assessed for $\alpha$-dystroglycan ( $\alpha$-DC), core-DC (45-3), and $\beta$-dystroglycan ( $\beta$-DC). Sarcoglycan antibodies utilized are specific for $\alpha$-sarcoglycan ( $\alpha$-SG), $\beta$-sarcoglycan ( $\beta$-SG), and $\gamma$-sarcoglycan $(\gamma$-SC). Scale bar: $50 \mu \mathrm{m}$. (B) Confocal immunofluorescence images of utrophin staining. Original magnification, $\times 63$. Scale bar: $100 \mu \mathrm{m}$. Confocal settings for gain and offset are the same for each antibody, as reported in the legend for Figure 4. (C) Intensity of sarcolemma protein staining of utrophin, Itgb, $\alpha$-DC (IIH6), and $\alpha$-SC is quantified from immunofluorescence images and all groups were compared by 1-way ANOVA using Tukey's multiple comparison test for each protein compared ( $n=3$ biological replicates, $n=6$ analyzed images). Data are presented as mean \pm SEM. Red dotted lines indicate WT values under identical exposure settings. $P \leq 0.05$ was considered significant. ${ }^{*} P \leq 0.05,{ }^{* *} P \leq 0.01,{ }^{* * *} P \leq 0.0001$. 
A

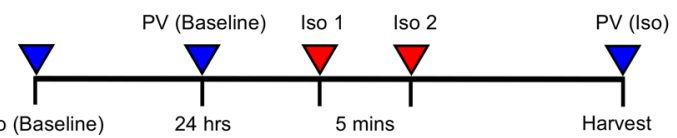

C

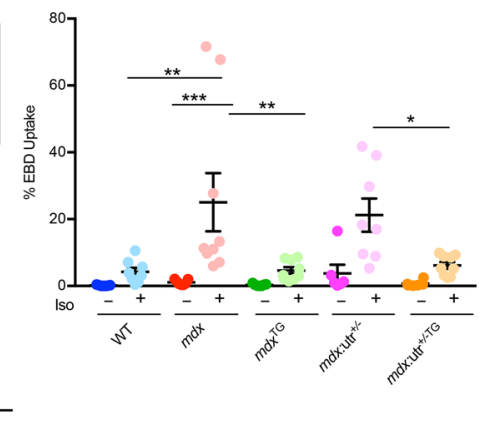

B

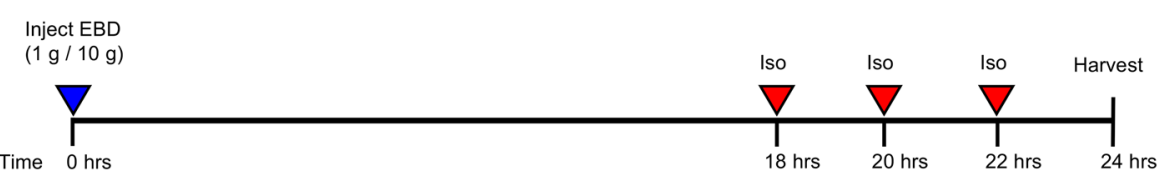

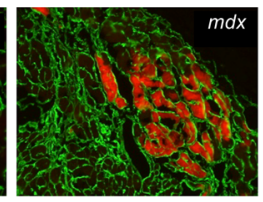
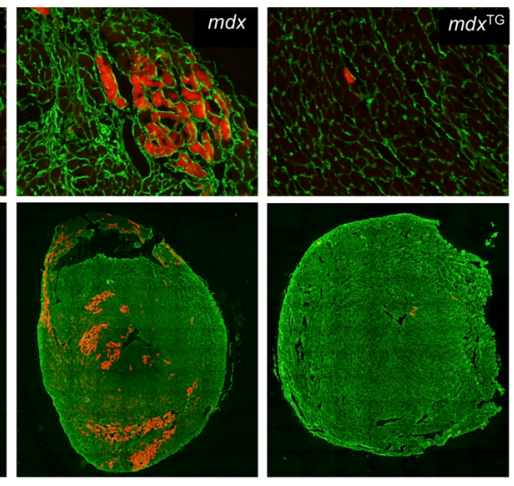
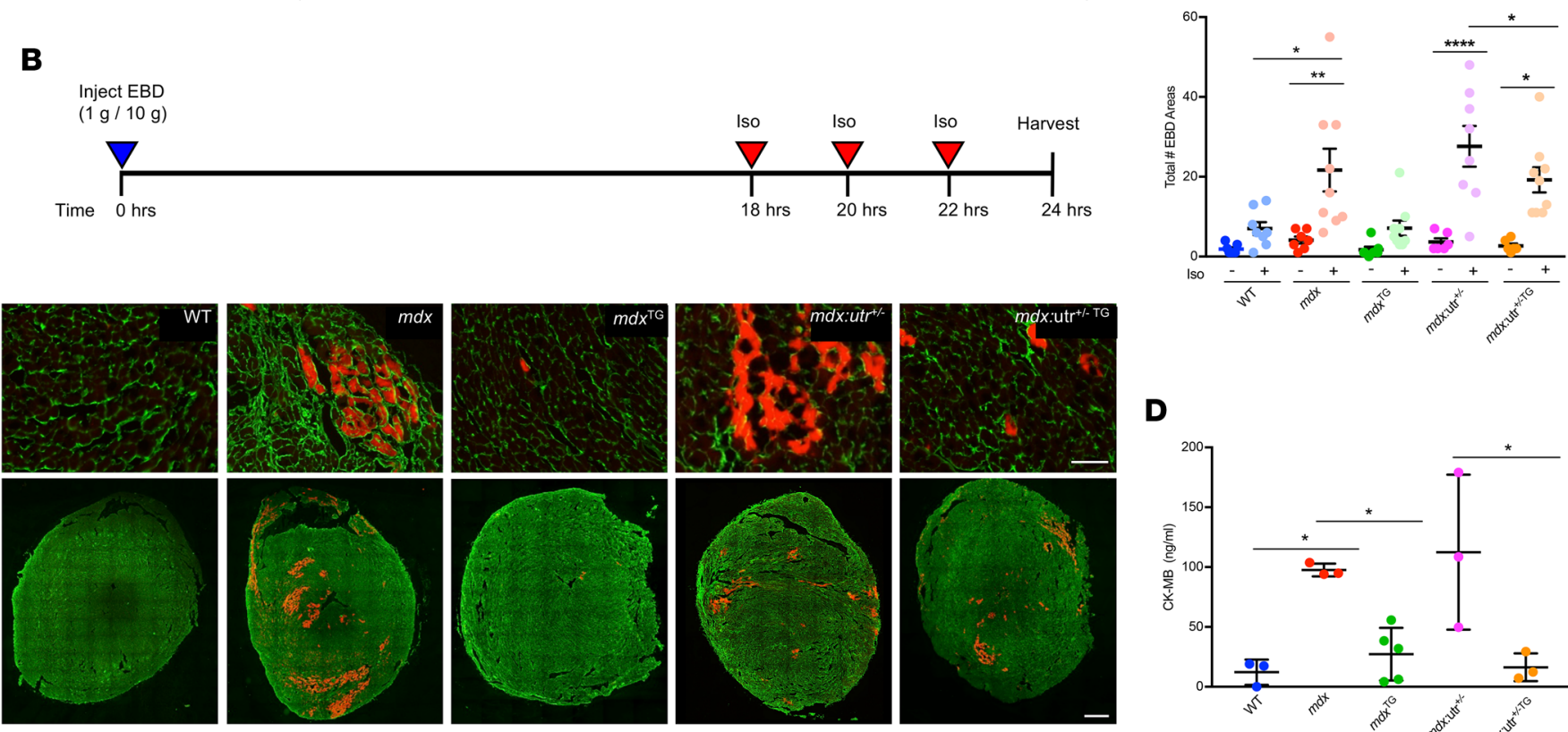

D

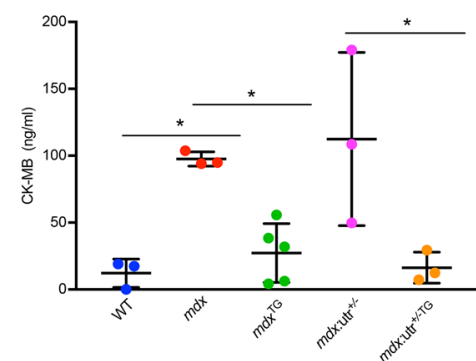

Figure 6. Sarcospan protects dystrophic hearts from $\boldsymbol{\beta}$-adrenergic-mediated membrane damage. (A) The diagram indicates the isoproterenol-dosing regimen utilized to test whether SSPN overexpression improves responsiveness to $\beta$-adrenergic stimulation. The immediate functional effects of $\beta$-adrenergic stimulation are reported on heart rate (HR), dP/dt max , and LV EF\% after 2 bolus doses of intravenous isoproterenol: Iso 1 indicates $30 \mathrm{ng} / \mathrm{kg}$ and Iso 2 indicates $90 \mathrm{ng} / \mathrm{kg}$ for the indicated genotypes. WT (untreated: $n=6$, post-Iso $n=6$ ), $m d x$ (untreated: $n=7$, post-Iso $n=7$ ), and $m d x^{\mathrm{TC}}$ (untreated: $n=7$, post-Iso $n=5$ ) mice were monitored before and after isoproterenol treatment by pressure-volume analysis. For WT LV EF\% values, measurements before and after isoproterenol were near significance: $P \leq 0.086$. Data are presented as individual points from each animal and are presented as mean \pm SEM. A summary of values is provided in Table 3. Individual Student's $t$ test compared the 2 groups (baseline versus isoproterenol-treated mice) for each

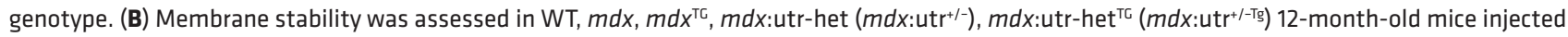
with Evan's blue dye (EBD; red fluorescence) and subjected to an isoproterenol-dosing regime and 3 intraperitoneal injections of 500 ng/g at 18-, 20-, and 22-hour time points. Transverse cardiac cryosections of isoproterenol-treated mice were costained with laminin antibodies (green fluorescence) and visualized by indirect immunofluorescence microscopy. Scale bar: $50 \mu \mathrm{m}$ (top); $900 \mu \mathrm{m}$ (bottom) ( $n=4$, each genotype). (C) Quantification of EBD uptake is reported in untreated (-) and isoproterenol-treated $(+)$ WT $(n=4), m d x(n=5), m d x^{\mathrm{TC}}(n=4), m d x$ :utr-het $\left(m d x:\right.$ utr $\left.^{+/-}\right)(n=4), m d x:$ utr-het $^{\mathrm{TC}}\left(m d x:\right.$ utr $\left.^{+/-\mathrm{Tg}_{\mathrm{g}}}\right)$ $(n=6)$ mice ( $n=2$ technical replicates). The percentage of EBD-positive area was calculated and reported for the above samples as a percentage relative to total tissue area (top). The total number of EBD-positive areas was also reported in the samples (bottom). Data are presented as individual points, and data are presented as mean \pm SEM. (D) Cardiac-specific creatine kinase (CK-MB) levels in the blood serum of 14-month-old untreated WT $(n=3), m d x(n=$ 3), $m d x^{\mathrm{TC}}(n=5), m d x: u t r-h e t\left(m d x: u^{+r^{+-}}\right)(n=3), m d x: u t r-$ het $^{\mathrm{TC}}\left(m d x: u t r^{+/-\mathrm{Tg}}\right)(n=3)$ mice. Results from each animal are plotted as individual points, and data are presented as mean \pm SEM. Data reported in $\mathbf{C}$ and $\mathbf{D}$ were compared by 1-way ANOVA utilizing Tukey's multiple comparison test. $P \leq 0.05$ was considered significant. ${ }^{*} P \leq 0.05,{ }^{* *} P \leq 0.01,{ }^{* *} P \leq 0.001,{ }^{* * *} P \leq 0.0001$.

for a DMD-specific antisense oligonucleotide therapy. Delivery of therapeutic modalities remains a leading source of difficulty in effectively treating DMD. The challenge remains significant; dystrophin is a major structural protein that must be reexpressed in a critical number of muscle fibers to positively effect heart, diaphragm, and skeletal muscle.

The current study examines the effectiveness of a membrane stabilization strategy to prevent the downstream cascade of deleterious effects resulting from loss of cell membrane integrity. In this study, we exploit the 
Table 3. Summary of pressure-volume studies in isoproterenol-challenged $m d x$ mice reveals a protective role for SSPN

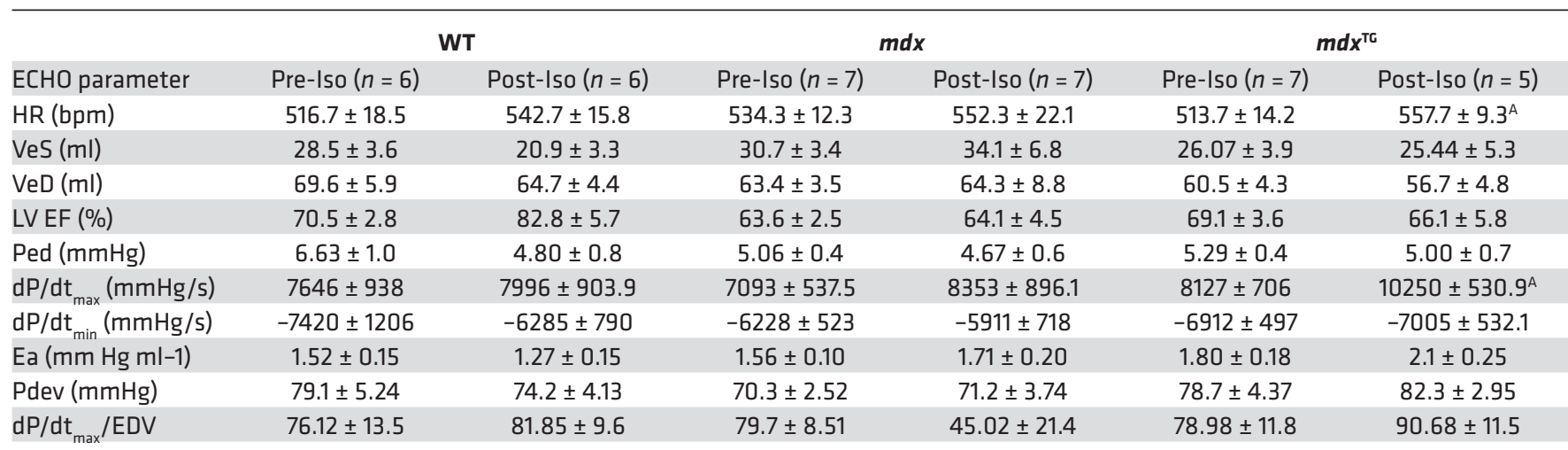

Genotypes analyzed include WT, dystrophin-deficient $m d x(m d x)$, and SSPN-Tg $m d x\left(m d x^{\mathrm{TC}}\right)$ mice. Baseline echo values (pre) and values after isoproterenol treatment (post) echo (Iso) are shown. Echo parameters include heart rate (HR); left ventricular (LV); end-systolic volume (VeS); enddiastolic volume (VeD); end-diastolic pressure (Ped); change in pressure over change in time during systole (dP/dt ${ }_{\max }$ ); change in pressure over change in time during diastole $\left(\mathrm{dP} / \mathrm{dt}_{\min }\right.$ ); arterial elastance $(E a)$; developed pressure (Pdev); change in pressure over change in time during systole as a function of end-diastolic volume ( $\left.\mathrm{dP} / \mathrm{dt}_{\max } / \mathrm{EDV}\right)$. Individual Student's $t$ test was used to determine differences between relevant groups before and after treatment with isoproterenol. ${ }^{A} P<0.05$ (comparison between $m d x^{\top \mathrm{C}}$ mice pre-Iso and post-Iso). Values are reported as averages (mean \pm SEM).

natural membrane-protective properties of SSPN, a protein member of the 3 major laminin-binding complexes in striated muscles $(5,14,15,34)$. In previous studies, we show that SSPN overexpression represents a promising avenue to prevent muscle membrane damage and subsequently improves baseline functional analysis of eccentric skeletal muscle contraction, pulmonary function, grip strength (31), and baseline cardiac function (30). In addition, our recent studies have shown that high levels of mouse SSPN overexpression are well tolerated and do not adversely affect health and life span (31). In the current study, we demonstrate that SSPN upregulation enhances sarcolemma distribution of utrophin without enhancing its abundance in $m d x$ hearts, which may be partially sufficient to protect cardiac tissue from damage and, thereby, decrease the extent of interstitial and focal fibrotic deposition. Previous studies have shown that Tg expression of utrophin in dystrophin-deficient models prevents DMD pathology and restores normal muscle function (38). Several factors appear important in the rescue of DMD by SSPN, including the ability to enhance utrophin and integrin expression that results in increased membrane stability and cellular adhesion in skeletal muscle $(34,39)$. These effects depend on activation of the Akt signaling cascade in skeletal muscle $(34,39)$. To determine the dependence of SSPN-mediated rescue upon utrophin expression, we asked whether the $m d x$ :utr-het mouse model with more severe/earlier onset cardiac dysfunction could be rescued with increased expression of SSPN. We examined the ability of SSPN overexpression to ameliorate both $m d x$ and $m d x$ :utr-het disease, in terms of cardiac function and membrane integrity defects. It was unknown, however, whether SSPN rescue of cardiac function and membrane fragility was dependent on a robust increase in utrophin expression, which has been documented (18) to compensate for dystrophin deficiency in DMD mouse models. In addition, it was uncertain whether SSPN overexpression exerted its protective influence by recruitment and stabilization of additional components (integrins and sarcoglycans) of the major adhesion complexes at the cell membrane without utrophin upregulation in DMD mouse models.

The findings from this study provide a mechanistic view of SSPN protection of dystrophic cardiac membranes and establish a basis for investigating a human SSPN-based therapeutic approach. We conclude that SSPN overexpression in $m d x$ mice, which exhibit a milder disease course, greatly reduced membrane defects revealed by the significant reduction of $\mathrm{EBD}$ uptake and serum CK-MB levels in $m d x^{\mathrm{TG}}$ and $m d x$ :utr-het ${ }^{\mathrm{TG}}$ mice versus relevant controls. A view of key sarcolemma adhesion complex proteins by immunofluorescence indicates that $m d x$ hearts have reduced $\beta 1 \mathrm{D}$ integrin, glycosylated $\alpha$-DG (IIH6), and sarcoglycan protein abundance compared with WT hearts. The DGC-associated proteins are largely retained in the heart and assembly of the complex does not depend on the presence of dystrophin $(21,37,40)$. In $m d x$ hearts, utrophin expression is measurably enhanced, exhibits a more stable sarcolemmal localization, and functions as a dystrophin surrogate (38). It is also hypothesized from studies examining dko (utrophin- and dystrophin-deficient) mice that continued DGC expression in $m d x$ hearts depends on utrophin sarcolemmal expression (41). In this study, overexpression of SSPN in $m d x$ hearts substantially increased $\beta 1 \mathrm{D}$ integrin abundance 
Table 4. Summary of membrane fragility studies in $m d x$ and $m d x$ :ur-het mice reveal that SSPN protects the sarcolemma during isoproterenol challenge

\begin{tabular}{|c|c|c|c|c|c|c|c|c|c|c|}
\hline $\begin{array}{l}\text { Measured } \\
\text { parameter }\end{array}$ & $\begin{array}{l}\text { WT Untr } \\
(n=4)\end{array}$ & $\begin{array}{l}\text { WT Iso } \\
(n=4)\end{array}$ & $\begin{array}{c}m d x \text { Untr } \\
(n=5)\end{array}$ & $\begin{array}{c}m d x \text { Iso } \\
(n=5)\end{array}$ & $\begin{array}{l}m d x^{\top \mathrm{TC}} \text { Untr } \\
\quad(n=4)\end{array}$ & $\begin{array}{c}m d x^{\mathrm{TC}} \text { Iso } \\
(n=4)\end{array}$ & $\begin{array}{l}\text { mdx:utr } \\
\text { Untr }(n=4)\end{array}$ & $\begin{array}{l}\text { mdx:utr }{ }^{+/-} \\
\text {Iso }(n=4)\end{array}$ & $\begin{array}{l}\text { mdx:utr' } / \text {-Tg } \\
\text { Untr }(n=6)\end{array}$ & $\begin{array}{l}\text { mdx:utr }{ }^{+/-T g} \\
\text { Iso }(n=6)\end{array}$ \\
\hline $\begin{array}{l}\text { EBD uptake } \\
(\%)\end{array}$ & $0.2 \pm 0.1$ & $4.3 \pm 1.2$ & $1.1 \pm 0.3$ & $25.1 \pm 8.7^{\mathrm{A}, \mathrm{B}}$ & $0.3 \pm 0.1$ & $4.6 \pm 1.0^{c}$ & $3.8 \pm 2.6$ & $21.2 \pm 5.0$ & $0.7 \pm 0.4$ & $6.2 \pm 1.0^{D}$ \\
\hline $\begin{array}{l}\text { No. EBD } \\
\text { areas }\end{array}$ & $1.9 \pm 0.5$ & $7.5 \pm 2.3$ & $4.1 \pm 0.9$ & $23.8 \pm 6.3^{\mathrm{A}, \mathrm{B}}$ & $1.7 \pm 0.8$ & $4.8 \pm 1.1$ & $3.7 \pm 0.9$ & $30.5 \pm 6.3^{A}$ & $2.7 \pm 0.6$ & $17.8 \pm 4.5^{A, D}$ \\
\hline $\begin{array}{l}\text { No. Lg. EBD } \\
\text { areas }\end{array}$ & - & $5.9 \pm 1.6$ & - & $14.0 \pm 3.9$ & - & $2.7 \pm 0.7$ & - & $21.5 \pm 4.2$ & - & $12.9 \pm 3.2$ \\
\hline $\begin{array}{l}\text { CK-MB } \\
(\mathrm{ng} / \mathrm{ml})\end{array}$ & $12.2 \pm 6.1^{\mathrm{E}}$ & - & $97.6 \pm 3.1^{\mathrm{F}}$ & - & $27.3 \pm 9.8$ & - & $112.4 \pm 37.4$ & - & $16.3 \pm 6.7^{\complement}$ & - \\
\hline
\end{tabular}

Values are from untreated (Untr) and isoproterenol-treated (Iso) mice; isoproterenol was administered (500 ng/g) at 18-, 20-, and 22-hour time points. Genotypes of mice include WT, dystrophin-deficient $m d x(m d x)$, SSPN-Tg $m d x\left(m d x^{\mathrm{TC}}\right)$, dystrophin-deficient $m d x$ with utrophin haploinsufficiency $\left(m d x: \mathrm{utr}^{+--}\right)$, and SSPN-Tg dystrophin-deficient $m d x$ with utrophin haploinsufficiency ( $m d x:$ utr $^{+/-T g}$ ) mice. EBD uptake (\%) represents the percentage of EBDpositive areas relative to total area measured per tissue sections obtained from isoproterenol-treated mice. The number of hearts examined per genotype and condition is shown. The number of EBD areas represents the number of EBD-positive areas measured per section. EBD-positive areas were measured and classified as small EBD areas if $>12,000 \mu \mathrm{m}^{2}$ and large EBD areas if $<12,000 \mu \mathrm{m}^{2}$. The CK-MB measurements report cardiac-specific creatine kinase measurements $(\mathrm{ng} / \mathrm{ml})$. One-way ANOVA was used to determine differences between relevant groups. ${ }^{A} P<0.05$ (comparison between untreated and isoproterenol-treated mice of same genotype), ${ }^{\mathrm{B}} P<0.05$ (comparison between WT and $\mathrm{mdx}$ isoproterenol-treated mice), ${ }^{\mathrm{C}} P<0.05$ (comparison between $m d x$ and $m d x^{\mathrm{TC}}$ isoproterenol-treated mice), ${ }^{\mathrm{D}} P<0.05$ (comparison between $m d x$ :utr ${ }^{+/-}$and $m d x$ : utr ${ }^{+/ \mathrm{Tg}}$ isoproterenol-treated mice), ${ }^{\mathrm{E}} P<0.05$ (comparison between WT and $m d x$ mice), ${ }^{\mathrm{F}} P<0.05$ (comparison between $m d x$ and $m d x^{\mathrm{TC}}$ mice), ${ }^{\mathrm{C}} P<0.05$ (comparison between $m d x$ :utr ${ }^{+/-}$and $m d x$ : utr ${ }^{+/-T g}$ mice), and ${ }^{\mathrm{H}} P$ $<0.05$ (comparison between number of small vs large EBD areas between mice of same genotype). Values are reported as averages (mean $\pm \mathrm{SEM}$ ).

and modestly increased glycosylated $\alpha$-DG; however, this occurred without a significant increase in utrophin levels. It appears that SSPN rescue of membrane instability was highly dependent on the stable expression of utrophin at the sarcolemma. Importantly, therapeutic levels of utrophin appeared lower than those found in WT muscle. Our data suggest that the most critical factor in utrophin-based modulation of DMD is the stable presence of utrophin at the sarcolemma, even at very low levels. Furthermore, we show that SSPN increased levels of $\beta 1 \mathrm{D}$ integrin and the sarcoglycans at the sarcolemma. An increase in $\alpha$-DG glycosylation may assist somewhat with membrane stability by improving cellular adhesion. In this regard, SSPN overexpression in $m d x$ :utr-het hearts modestly increased abundance of these proteins at the sarcolemma, though not to the same extent as in $m d x$ hearts. $\beta 1 \mathrm{D}$ integrin, sarcoglycans, and glycosylated $\alpha$-DG levels were also enhanced in $m d x$ :utr-het ${ }^{\mathrm{TG}}$ hearts, indicating that the effect of SSPN on boosting the expression of these proteins was not utrophin dependent. The $m d x$ :utr-het hearts displayed even lower levels of associated proteins, including the $\alpha 7 / \beta 1 \mathrm{D}$ integrin complex than $m d x$ hearts; however, these proteins were still substantially increased by SSPN overexpression. Interestingly, the core DG protein levels appeared similar in WT and SSPN-overexpressing hearts on either $m d x$ or $m d x$ : utr-het backgrounds. The quantity of SSPN appears to have an important influence on $\alpha$-DG glycosylation. From this study, it can be concluded that SSPN overexpression robustly enhances stable expression of adhesion complex proteins and $\alpha$-DG glycosylation at the cardiac cell membrane.

The next fundamental question addressed was whether the small enhancement in sarcolemma protein abundance in $m d x$ :utr-het ${ }^{\mathrm{TG}}$ hearts compared with control was sufficient to preserve cardiac membrane integrity under stressed ( $\beta$-adrenergic stimulation) and nonstressed conditions. Overall, $m d x$ hearts do not exhibit the same degree of membrane damage (reported as EBD uptake) as skeletal muscle, likely due to retention of the DGC protein complex and smaller cell sizes in cardiac muscle. Administration of an isoproterenol-dosing regimen after EBD injection was intended to increase cardiac contractility and exacerbate membrane defects in dystrophic hearts. As expected only low levels of cardiac membrane damage were present in WT hearts after isoproterenol treatment. It has been shown by targeted tissue-specific deletion in mice that $\alpha$-DG disruption in cardiac or vascular tissue causes accumulation of clusters of membrane damage, leading to formation of large areas of membrane damage in cardiac cell membranes. From this study it could be concluded that the extracellular matrix receptor role of $\alpha$-DG is crucial for preventing spreading of damage to adjacent cardiomyocytes during conditions of increased load (42). 
In the current study, the membrane-stabilizing role of SSPN was assessed; however, it is also evident that reduction in functional $\alpha$-DG (IIH6 staining) and not $\alpha$-DG protein (core-DG) levels in dystrophin-deficient hearts may underlie the significant reduction in $m d x$ :utr-het cardiac membrane stability compared with that in $m d x$ hearts. Both, $m d x$ and $m d x$ :utr-het hearts exhibited many EBD-positive areas after isoproterenol treatment, indicating that damage-spreading mechanisms attributed to the initial weakening of cardiomyocyte cell membranes, which in turn may affect membrane integrity of neighboring cardiomyocytes. Significantly, there was very little EBD uptake in $m d x^{\mathrm{TG}}$ hearts and much reduced EBD uptake in $m d x$ :utr-het ${ }^{\mathrm{TG}}$ hearts compared with NTG controls. This may be potentially due to a 2-fold mechanism of cell membrane protection that can be attributed to SSPN, which increases expression of proteins, which strengthens the lateral (sarcoglycans) and vertical axes (DG proteins and glycosylation status) governing membrane stability. Dependence of this protection on SSPN can be seen in costained SSPN-EBD cardiac sections, which indicate that EBD-positive areas frequently correspond to regions of low SSPN expression. When examining isoproterenol-mediated effects on EBD uptake, the potential for confounding effects exists, since dystrophin deficiency renders $m d x$ mice less responsive to $\beta$-adrenergic stimulation, whereas SSPN overexpression improves some aspects of responsiveness. Analysis of membrane integrity defects by examining release of cardiac-specific CK-MB isoform into blood serum revealed the same trends as shown by the EBD uptake measurements.

The stabilization of cardiac cell membranes improved baseline cardiac function in $m d x$ mice overexpressing low levels of SSPN (30). Here, the effects of high levels of SSPN expression were comprehensively examined in terms of cardiac performance. It was anticipated that targeting cell membrane defects addresses the primary structural defect in DMD and may subsequently prevent many downstream pathogenic effects that drive progression of dystrophic cardiac disease. Furthermore, analysis of SSPN rescue of cardiac function was examined in the context of mild and severe dystrophic disease states to facilitate analysis of functional rescue and more closely approximate human disease mechanisms. Overall, SSPN overexpression improved aspects of pathogenic ventricular remodeling, whereas $m d x$ :utr-het mice exhibited a more advanced disease stage compared with $m d x$ mice, therefore providing greater capacity for correction. In contrast to the improvement in diastolic parameters attributed to poloxamer 188 administration in golden retriever muscular dystrophy dogs and $m d x$ mice $(43,44)$, SSPN overexpression improved systolic function and ventricular geometries of $m d x$ and $m d x$ :utr-het mice characterized by a significant reduction in LVESD. Furthermore, overexpression of SSPN in $m d x$ mice restored contractility, shown by increased LV EF\% and LV FS\% values that were close to WT (C57BL6/J) values under similar conditions (45); however, this effect was dampened in $m d x$ :utr-het mice. Interestingly, LVEDD was significantly improved in $m d x$ :utr-het mice by SSPN overexpression, potentially because $m d x$ :utr-het mice initially exhibited more advanced pathological cardiac remodeling. Therefore, in the spectrum of DMD disease progression, it appears that enhanced SSPN expression may preserve systolic function. Furthermore, a decline in systolic function marks the final stage of DMD cardiomyopathy prior to the development of end-stage heart failure. Diastolic dysfunction occurs during early disease stages of DMD cardiac dysfunction; however, SSPN overexpression does not appear to significantly correct dysfunction at this stage. The effect of SSPN in ameliorating DMD-associated cardiomyopathy may be its ability to preserve systolic function and subsequently delay progression toward heart failure.

Cardiac function of $m d x$ and $m d x^{\mathrm{TG}}$ mice was assessed near the peak of disease, beyond the 10-month mark, when $m d x$ mice are documented to exhibit discernible cardiac dysfunction. Early stages of DMD-associated cardiomyopathy manifest as diastolic dysfunction in patients (46). Since $m d x$ mice exhibit subtle cardiac dysfunction at 12 months of age, the benefits of SSPN overexpression were examined in further depth using hemodynamic pressure-volume analysis. Overall, improvement was seen in systolic function of $m d x^{\mathrm{TG}}$ mice compared with $m d x$ mice, indicating an overall increase in cardiac contractility. The output work capacity of the cardiac muscle reported by $\mathrm{CO}$ and SV was not substantially improved in $m d x^{\mathrm{TG}}$ hearts, although the SW was significantly improved, perhaps because of increased vascular resistance that may contribute to improved ventricular pressures during systole. A more precise view of ventricular function can be gathered from functional measurements that report intrinsic properties of the heart, including preload recruitable SW, but also provide insight on the influence of the vasculature on the heart performance, including SV, CO, and the maximum pressure derivative $\left(\mathrm{dP} / \mathrm{dt}_{\max }\right)$.

Previous studies have revealed that $m d x$ mice exhibit blunted responsiveness to $\beta$-adrenergic stimulation, partially attributed to increased myocyte loss and reduction in cardiac reserve (47). Responsiveness to $\beta$-adrenergic stimulation is reduced in $m d x$ mice at early stages of cardiac dysfunction and initial increases in contractile function compensate for myocyte loss. However, myocardial fibrosis begins to occur by 2 


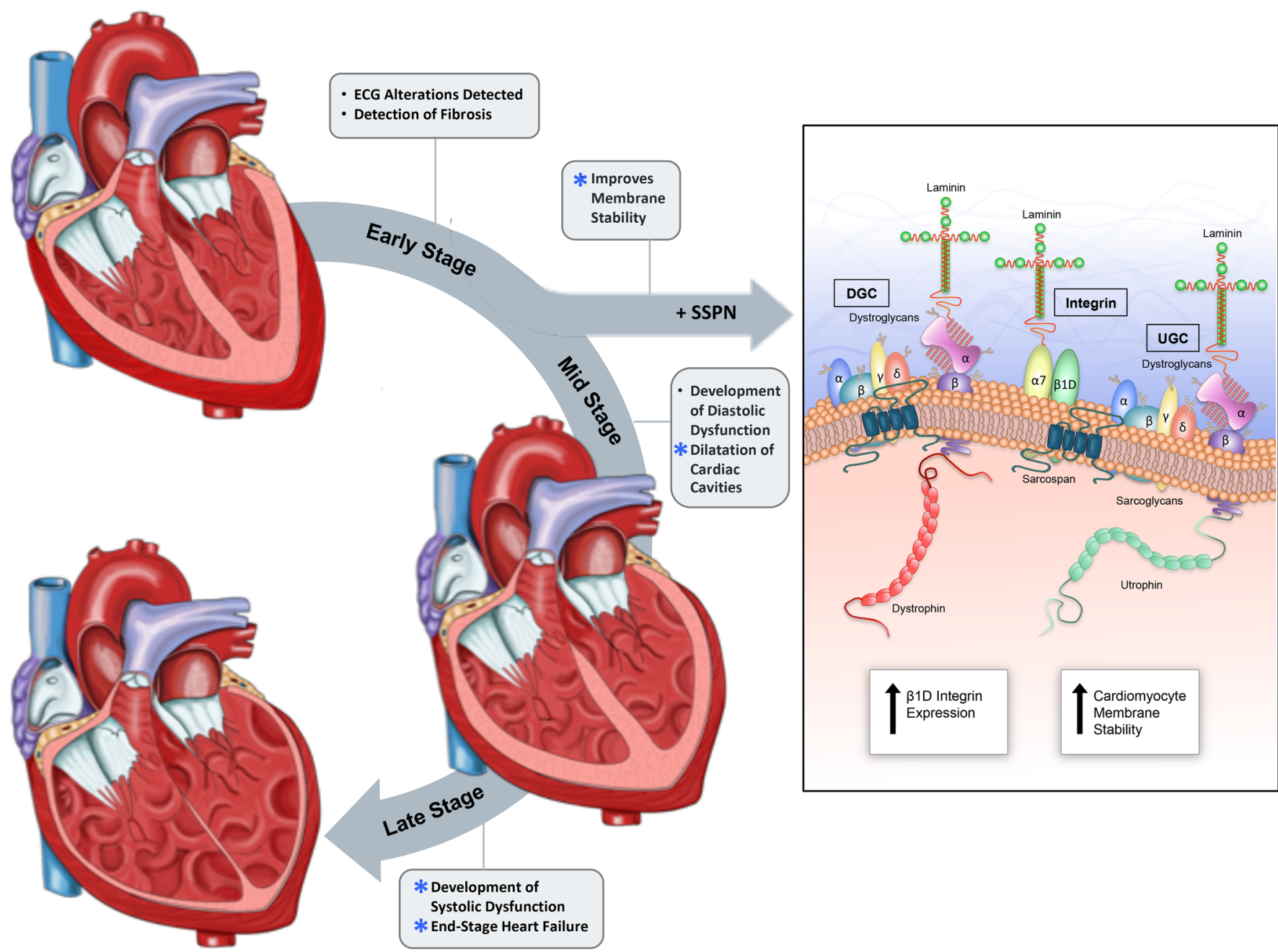

Figure 7. Sarcospan overexpression provides system-wide correction of DMD muscle pathology. A schematic illustration depicts the major therapeutic benefits of SSPN overexpression in DMD-associated cardiomyopathy based on our preclinical studies in several relevant DMD murine models. Areas of improvement in the DMD-associated cardiomyopathy disease course are marked with blue asterisks. SSPN overexpression has many beneficial effects, as highlighted, including enhancing systolic function and prevention of ventricular remodeling. These positive outcomes are mediated primarily by stabilizing the cell membrane subsequently protecting against cardiomyocyte loss and downstream consequences, including fibrosis and cardiac dysfunction. A schematic of the protein complexes affected by SSPN overexpression is also shown, which protects the myocardium. Improvements in cell membrane stability result in lower EBD uptake in cardiac muscle, reduced muscle/cardiac creatine kinase leakage into the blood, and decreased fibrosis. The overall ability of SSPN to increase abundance of $\beta 1 D$ integrin, sarcoglycans, dystroglycans and perhaps utrophin enhance cardiomyocyte sarcolemma stability and protect against functional decline and tissue damage.

months of age in $m d x$ mice, 6 months before cardiac remodeling and dysfunction become evident (47). This baseline increase in function reduces the stimulatory potential of the heart, which can also be attributed to a reduction in cardiac reserve. In this study, we examined whether SSPN overexpression restores in any way the heart's capacity to respond to $\beta$-adrenergic stimulation. The acute effects of isoproterenol administration were tested using 2 bolus doses and monitored by pressure-volume analysis. Immediate effects on heart rate and $\mathrm{dP} / \mathrm{dt}_{\max }$ could be detected in $m d x^{\mathrm{TG}}$ mice after isoproterenol administration, which were absent in $m d x$ mice. This indicates that SSPN was able to restore some degree of functional responsiveness in $m d x$ mice that normally exhibit a blunted cardiac $\beta$-adrenergic response.

The schematic included in Figure 7 summarizes the major outcomes of our preclinical studies, establishing the safety and efficacy of SSPN overexpression as a DMD therapeutic strategy. In this and previous studies, SSPN has been demonstrated to unequivocally improve skeletal $(5,31-32)$, pulmonary (31), and cardiac pathology and function (30) in DMD mouse models. The schematic in Figure 7 shows effects of SSPN overexpression in $m d x^{\mathrm{TG}}$ and $m d x$ : utr-het ${ }^{\mathrm{TG}}$ hearts. At the molecular level, SSPN upregulates $\beta 1 \mathrm{D}$ integrin and, together with the enhanced SSPN-Tg subcomplex at the sarcolemma, can 
partially compensate for the absence of dystrophin. Since utrophin is not upregulated to any significant level, this study reveals that SSPN amelioration of DMD cardiac failure is not dependent on increased utrophin abundance but rather its stable localization at the cardiac sarcolemma. An increase in utrophin may further accentuate the effectiveness of SSPN rescue; however, we found that it is not required. The increase in retention or recruitment of SSPN-associated proteins ( $\beta 1 \mathrm{D}$ integrin and SGs) by SSPN overexpression was increased in $m d x$ hearts compared with $m d x$ :utr-het hearts. It is unknown whether the amount of utrophin normally upregulated in $m d x$ hearts benefited SSPN-mediated rescue of $m d x$ hearts compared with $m d x$ :utr-het hearts, which had much lower utrophin expression. The presence of more utrophin in $m d x^{\mathrm{TG}}$ hearts may have allowed SSPN to better recruit or retain sarcolemma membrane-stabilizing complexes relative to the $m d x$ :utr-het ${ }^{\mathrm{TG}}$ hearts. Higher baseline utrophin levels may contribute to, but not be the ultimate source of, the enhanced rescue by SSPN in $m d x$ versus $m d x$ : utr-het hearts. The studies in the $m d x$ :utr-het background, which had low background utrophin expression, confirmed that SSPN rescue was not dependent on utrophin. Further cell membrane stabilization appears to be derived by effects of SSPN on $\alpha$-DG glycosylation. SSPN overexpression enhances $\alpha$-DG glycosylation, thereby enhancing cellular adhesion, by increasing binding to laminin in the extracellular matrix. Therefore, the ameliorative effects of SSPN can be attributed to its stabilizing role on the cell membranes of both skeletal and cardiac muscles. Correction of the primary defect in DMD, loss of cell membrane integrity, appears beneficial in preserving muscle integrity and function and protects skeletal, diaphragm, cardiac muscle integrity, and function from downstream pathological consequences that occur as a result of muscle cell membrane injury. In Figure 7, the normal disease course of DMD-associated cardiomyopathy is shown, highlighting the stage of disease progression that SSPN overexpression addresses. Importantly, overexpression of SSPN appears to slow the progression of cardiomyopathy in DMD mice and prevents development of systolic dysfunction and subsequently the development of heart failure.

In summary, SSPN overexpression improves cardiomyocyte membrane integrity, allowing both $m d x$ and $m d x$ :utr-het hearts to better withstand mechanical sheer stresses placed on cell membranes. The improvement in membrane stability limits the number and size of membrane lesions, which, in turns, reduces strain placed on the undamaged myocardium. This, in turn, lessens necrotic cell death, resulting fibrosis, and thereby reduces cardiac cell wall remodeling and ventricular dilation during the course of DMD cardiomyopathy disease progression. We demonstrate that correcting membrane permeability defects improves cardiac function in both mild and severe DMD models and partially restores $\beta$-adrenergic responsiveness to $m d x$ hearts. Furthermore, we show that SSPN overexpression is able to ameliorate cardiac function and membrane integrity without upregulating utrophin, therefore showing promise in alleviating both skeletal and cardiac muscle dysfunction in human disease.

\section{Methods}

\section{Animal models}

SSPN (line 28) Tg mice were generated previously using standard procedures (31). The murine SSPN-Tg is expressed in skeletal and cardiac muscles under control of the human skeletal actin promoter (48). WT C57BL6/J and $m d x$ mouse strain C57BL/10ScSn-Dmd $m d x / \mathrm{J}$ were obtained from Jackson Laboratories. The SSPN-Tg mice used in this study were maintained on the C57BL/10ScSn-Dmd $m d x / \mathrm{J}$ background and were produced with the following crosses: $m d x^{\mathrm{TG}}$ mice were produced using $m d x^{\mathrm{TG}}$ male $\times m d x$ female crosses, and $m d x$ :utr-het ${ }^{\mathrm{TG}}$ mice were produced using $m d x^{\mathrm{TG}}$ male $\times m d x$ :utr-het female crosses. NTG ( $m d x^{\mathrm{NTG}}$ and $m d x$ :utr-het ${ }^{\mathrm{NTG}}$ ) littermates were used as controls. The $m d x$ :utr-het mice were obtained in-house and were derived from the colony originally generated by Joshua R. Sanes (Washington University School of Medicine, St. Louis, Missouri, USA). The mice were genotyped using published protocols $(48,49)$. Mice were maintained in the Terasaki Life Sciences Vivarium.

\section{AAV construct cloning, vector production, and administration}

Construction. Recombinant adeno-associated viral (rAAV) vector genomes containing an expression cassette for SSPN were generated using standard cloning techniques (50). EagI fragments of the coding sequence for microdystrophin were blunted with T4 DNA polymerase and cloned into the plasmid ARAP4 after HindIII digestion and blunting of the vector plasmid. The CMV promoter was introduced into this construct by ligating a MluI-blunted/SacII fragment of pCMV-MCS (Stratagene) into the XbaI-blunted/SacII site of the plasmid. 
Vector production. The resulting construct was cotransfected with the packaging plasmid (pDGM6) into HEK293 cells to generate rAAV vectors with serotype 6 capsids. The rAAV6 vectors were harvested, enriched, and quantified as previously described (51). Enriched preparations (up to $\sim 1 \times 10^{14}$ vector genomes $/ \mathrm{ml}$ ) of $\mathrm{rAAV} 6$ vectors were stored at $-80^{\circ} \mathrm{C}$ in phosphate-buffered saline until thawed for use (52).

Administration. The rAAV6-SSPN constructs were administered to 2 -week-old pups by retro-orbital administration of $1.6 \times 10^{12}$ vector genomes, the hearts were collected at 6 weeks of age $(n=4)$.

\section{Histological and immunofluorescence assays}

$\mathrm{H} \& \mathrm{E}$ staining was used to visualize changes in tissue architecture, including myocyte disarray, hypertrophy, and fibrosis. Masson's trichrome staining was utilized to detect collagen deposition. To determine the amount of fibrosis, the fibrotic area was measured using ImageJ 1.50i software (NIH freeware) and compared with total area of transverse cardiac sections. Immunofluorescence assays were performed and visualized as described previously (5). Cardiac section images were examined using a confocal fluorescence imaging microscope (Leica TCS-SP5) at the Advanced Light Microscopy Imaging Core at UCLA. The following antibodies were used: dystrophin (MANDYS1) (1:5; Development Studies Hybridoma Bank); utrophin (MANCHO3) (1:5; Development Studies Hybridoma Bank); $\alpha$-DG IIH6 (sc-53987) (1:500; Santa Cruz Biotechnology Inc.); $\alpha$-DG core (45-3, 1:5 serum-free fraction) (53); $\beta$-DG (MANDAG2) (1:50; Development Studies Hybridoma Bank); $\alpha$-SG (VP-A105) (1:30; Vector Labs); $\beta$-SG (VP-B206); ( $\gamma$-SG (VP-G803) (1:30; Vector Labs); $\beta 1 D$ integrin (MAB1900) (1:20; Chemicon); SSPN E-2 (sc-393187) (1:25; Santa Cruz Biotechnology Inc.); and laminin anti-rabbit (L9393 SIGMA) (1:25; Millipore Sigma). Primary antibodies were detected using biotinylated anti-mouse antibody (BA9200) (1:500; Vector Labs) and biotinylated anti-rabbit (BA-1000) (1:500; Vector Labs) for detection of laminin primary antibody. Fluorescein-conjugated (A-2001) (1:500; Vector Labs) avidin D was used to detect biotinylated secondary antibodies. Samples tagged with FITC fluorophores were excited using a high-powered argon laser and sequentially imaged at $\times 20$ and $\times 63$ magnification to explore localization and abundance of various DGC and UGC components. The microscope is a confocal fluorescence imaging microscope (Leica TCS-SP5) with Argon laser ( $65 \mathrm{~mW}$; 458, 476, 488, 496, $514 \mathrm{~nm}$ ). Exposure times were kept the same between compared groups, as indicated in figure legends. Images were visualized in real-time 3D with Leica Application Suite Advanced Fluorescence software, where system parameters and data acquisition can be applied, analyzed, and processed for data collection.

\section{EBD uptake and isoproterenol challenge}

Sarcolemmal membrane damage was assessed using EBD tracer analysis as described previously (39). EBD was administered at time 0 and challenged with isoproterenol (500 ng/g) at 18-, 20-, and 22-hour time points and dissected 24 hours after EBD injection. Cardiac muscle was mounted in $10.2 \%$ polyvinyl alcohol/4.3\% polyethylene glycol and flash-frozen in liquid nitrogen-cooled isopentane. The percentage of EBD-positive cardiac tissue was obtained using ImageJ $1.50 \mathrm{i}$ software to measure EBD-positive areas in transverse whole heart sections divided by total area. EBD fluoresces red at excitation wavelength $620 \mathrm{~nm}$. Sections were counterstained with 1:25 dilution of rabbit anti-laminin primary antibodies (Millipore Sigma, L9393) and 1:500 dilution of Cy5 (FITC-conjugated) goat anti-rabbit IgG secondary antibody (Jackson Immunoresearch, 111-175-144) to outline sarcolemma boundaries.

\section{Creatine kinase assay (CK-MB)}

CK-MB measurements were conducted using the LSBio Inc. Mouse CK-MB/Creatine Kinase MB ELISA Kit (Sandwich ELISA) (LS-F5745) according to the manufacturer's directions.

\section{Echocardiography}

Left ventricular size, mass, wall thickness, ventricular and valve function, and Doppler blood flows were obtained using previously described methods $(54,55)$. Briefly, mice were anesthetized with isoflurane $(1 \%-3 \%)$ and body temperature maintained at $37^{\circ} \mathrm{C}$. Paraesternal long-axis and short-axis images were obtained to determine cardiac morphology and function. The ratio of the left ventricular transmitral early peak flow (E wave) to late peak flow (A wave) velocity (E/A ratio) was determined for diastolic function evaluation. All echocardiographic assessments were analyzed offline (Vevo LAB 2.1.0 software, Visual Sonics) over 3-5 consecutive heartbeats. 


\section{Hemodynamic cardiac measurements}

Hemodynamic assessments were performed using a microtipped pressure-volume catheter (Millar Instruments, SPR-839) as previously described with minor modifications (54). Briefly, mice were anesthetized with isoflurane $(2 \%-3 \%)$ before the catheter was inserted into the right carotid artery and advanced retrograde into the left ventricle. Pressure-volume loops were recorded at steady state during temporary inferior vena cava occlusion and after isoproterenol bolus administration via jugular vein (bolus $1: 30 \mathrm{ng} / \mathrm{kg}$; bolus 2: $90 \mathrm{ng} / \mathrm{kg}$ ). All analyses were performed using LabChart Pro version 8.1 .5 software (AD Instruments).

\section{Statistics}

All values in the text and figures are presented as mean \pm SEM, unless otherwise indicated. Statistical significance was determined using a 2-tailed Student's $t$ test to compare relevant groups of 2 (e.g., untreated versus treated) or to compare differences within an age-matched specific DMD phenotype in NTG versus Tg mice. Ordinary 1-way ANOVA was used when comparisons were made across all groups, followed by Tukey's multiple comparisons test. $P<0.05$ was considered significant.

\section{Study approval}

The animal studies included in this study were reviewed and approved by the Institutional Animal Care and Use Committee at the UCLA and at Florida State University.

\section{Author contributions}

MSP designed and conceived of the study, generated mice, performed data analysis, supervised and participated in data collection, prepared figures, and drafted the manuscript. AJB performed data analysis, prepared figures, and performed immunofluorescence staining, imaging, and histology. RMKT performed PV loop experiments; provided expertise in data analysis, interpretation, and echocardiography; and reviewed the manuscript. KMDJ performed baseline echocardiography on all mice, analyzed data, and helped with the EBD experimental protocol. JRC and KGH performed fluorescence and confocal imaging, fibrosis quantification, histological staining, and immunofluorescence. KGH created schematic illustrations. JG performed immunofluorescence staining, imaging, and qRT-PCR analysis. AF characterized mice and performed immunofluorescence staining, imaging, and histology. JLM created and designed SSPN-Tg mice and AAV6-SSPN. AMB generated and provided the core dystroglycan antibody. JSC designed and produced AAV6 and provided AAV expertise. JRP designed the study, supervised studies at the Florida State University site, provided ultrasound and PV loop expertise and interpretation as well as project support during data collection, and reviewed the manuscript. RHC designed and conceived of the study, supervised the study at the UCLA site, provided project support and manuscript preparation as well as data review and oversight.

\section{Acknowledgments}

We thank Elizabeth Gibbs for assistance with AAV experiments. Funding support was generously provided by the Center for Duchenne Muscular Dystrophy-CureDuchenne Postdoctoral Fellowship and American Heart Association Scientist Development grant 16SDG29120002 to MSP; the UCLA CARE Fellowship to JRC; NIH grants T34 GM008563 and R25 GM050067 to AF; NIH grant HL128683 to JRP; NIH grant F31 HL137408 to KMDJ; NIH grant R01 HL122332 to JSC; and NIH grants AR048179, HL126204, UL1TR000124, 5P30AR057230 (pilot and feasibility), and MDA 274143 funding support to RHC.

Address correspondence to: Rachelle H. Crosbie, Department of Integrative Biology and Physiology, Department of Neurology, David Geffen School of Medicine at UCLA, University of California, Los Angeles, Center for Duchenne Muscular Dystrophy, 610 Charles E. Young Drive East, Terasaki Life Sciences Building Room 1121, Los Angeles, California 90095, USA. Phone: 310.794.2103; Email: rcrosbie@physci.ucla.edu.

MSP's present address is: Department of Nutrition, Food and Exercise Sciences, Florida State University, Tallahassee, Florida, USA.

1. Emery AE. Population frequencies of inherited neuromuscular diseases--a world survey. Neuromuscul Disord. 1991;1(1):19-29.

2. Romitti PA, et al. Prevalence of Duchenne and Becker muscular dystrophies in the United States. Pediatrics. 2015;135(3):513-521.

3. Talsness DM, Belanto JJ, Ervasti JM. Disease-proportional proteasomal degradation of missense dystrophins. Proc Natl Acad Sci 
USA. 2015;112(40):12414-12419.

4. Briguet A, et al. Effect of calpain and proteasome inhibition on $\mathrm{Ca} 2+$-dependent proteolysis and muscle histopathology in the mdx mouse. FASEB J. 2008;22(12):4190-4200.

5. Marshall JL, Chou E, Oh J, Kwok A, Burkin DJ, Crosbie-Watson RH. Dystrophin and utrophin expression require sarcospan: loss of $\alpha 7$ integrin exacerbates a newly discovered muscle phenotype in sarcospan-null mice. Hum Mol Genet. 2012;21(20):4378-4393.

6. Lapidos KA, Kakkar R, McNally EM. The dystrophin glycoprotein complex: signaling strength and integrity for the sarcolemma. Circ Res. 2004;94(8):1023-1031.

7. Finsterer J, Cripe L. Treatment of dystrophin cardiomyopathies. Nat Rev Cardiol. 2014;11(3):168-179.

8. Lim KR, Maruyama R, Yokota T. Eteplirsen in the treatment of Duchenne muscular dystrophy. Drug Des Devel Ther. 2017;11:533-545.

9. Spurney CF. Cardiomyopathy of Duchenne muscular dystrophy: current understanding and future directions. Muscle Nerve. 2011;44(1):8-19.

10. Campbell KP, Kahl SD. Association of dystrophin and an integral membrane glycoprotein. Nature. 1989;338(6212):259-262

11. Ervasti JM, Kahl SD, Campbell KP. Purification of dystrophin from skeletal muscle. J Biol Chem. 1991;266(14):9161-9165.

12. Ervasti JM, Ohlendieck K, Kahl SD, Gaver MG, Campbell KP. Deficiency of a glycoprotein component of the dystrophin complex in dystrophic muscle. Nature. 1990;345(6273):315-319.

13. Ervasti JM, Campbell KP. Membrane organization of the dystrophin-glycoprotein complex. Cell. 1991;66(6):1121-1131.

14. Crosbie RH, Heighway J, Venzke DP, Lee JC, Campbell KP. Sarcospan, the 25-kDa transmembrane component of the dystrophin-glycoprotein complex. J Biol Chem. 1997;272(50):31221-31224.

15. Crosbie RH, et al. Membrane targeting and stabilization of sarcospan is mediated by the sarcoglycan subcomplex. $J$ Cell Biol. 1999;145(1):153-165.

16. Crosbie RH, et al. Molecular and genetic characterization of sarcospan: insights into sarcoglycan-sarcospan interactions. Hum Mol Genet. 2000;9(13):2019-2027.

17. Miller G, Wang EL, Nassar KL, Peter AK, Crosbie RH. Structural and functional analysis of the sarcoglycan-sarcospan subcomplex. Exp Cell Res. 2007;313(4):639-651.

18. Tinsley J, et al. Expression of full-length utrophin prevents muscular dystrophy in mdx mice. Nat Med. 1998;4(12):1441-1444.

19. Jasmin BJ, et al. Multiple regulatory events controlling the expression and localization of utrophin in skeletal muscle fibers: insights into a therapeutic strategy for Duchenne muscular dystrophy. J Physiol Paris. 2002;96(1-2):31-42.

20. Pons F, et al. Utrophin localization in normal and dystrophin-deficient heart. Circulation. 1994;90(1):369-374.

21. Matsumura K, Ervasti JM, Ohlendieck K, Kahl SD, Campbell KP. Association of dystrophin-related protein with dystrophin-associated proteins in mdx mouse muscle. Nature. 1992;360(6404):588-591.

22. Clerk A, Morris GE, Dubowitz V, Davies KE, Sewry CA. Dystrophin-related protein, utrophin, in normal and dystrophic human fetal skeletal muscle. Histochem J. 1993;25(8):554-561.

23. Lin S, Burgunder JM. Utrophin may be a precursor of dystrophin during skeletal muscle development. Brain Res Dev Brain Res. 2000;119(2):289-295

24. Miura P, Jasmin BJ. Utrophin upregulation for treating Duchenne or Becker muscular dystrophy: how close are we? Trends Mol Med. 2006;12(3):122-129.

25. Tinsley JM, et al. Daily treatment with SMTC1100, a novel small molecule utrophin upregulator, dramatically reduces the dystrophic symptoms in the mdx mouse. PLoS One. 2011;6(5):e19189.

26. Krag TO, et al. Heregulin ameliorates the dystrophic phenotype in mdx mice. Proc Natl Acad Sci USA. 2004;101(38):13856-13860.

27. Deconinck AE, et al. Utrophin-dystrophin-deficient mice as a model for Duchenne muscular dystrophy. Cell. 1997;90(4):717-727.

28. Janssen PM, Hiranandani N, Mays TA, Rafael-Fortney JA. Utrophin deficiency worsens cardiac contractile dysfunction present in dystrophin-deficient mdx mice. Am J Physiol Heart Circ Physiol. 2005;289(6):H2373-H2378.

29. van Putten M, et al. Comparison of skeletal muscle pathology and motor function of dystrophin and utrophin deficient mouse strains. Neuromuscul Disord. 2012;22(5):406-417.

30. Parvatiyar MS, et al. Sarcospan Regulates Cardiac Isoproterenol Response and Prevents Duchenne Muscular Dystrophy-Associated Cardiomyopathy. J Am Heart Assoc. 2015;4(12):null.

31. Gibbs EM, et al. High levels of sarcospan are well tolerated and act as a sarcolemmal stabilizer to address skeletal muscle and pulmonary dysfunction in DMD. Hum Mol Genet. 2016;25(24):5395-5406.

32. Peter AK, Marshall JL, Crosbie RH. Sarcospan reduces dystrophic pathology: stabilization of the utrophin-glycoprotein complex. J Cell Biol. 2008;183(3):419-427.

33. Marshall JL, et al. Sarcospan integration into laminin-binding adhesion complexes that ameliorate muscular dystrophy requires utrophin and $\alpha 7$ integrin. Hum Mol Genet. 2015;24(7):2011-2022.

34. Marshall JL, et al. Sarcospan-dependent Akt activation is required for utrophin expression and muscle regeneration. J Cell Biol. 2012;197(7):1009-1027.

35. Rader EP, et al. Role of dystroglycan in limiting contraction-induced injury to the sarcomeric cytoskeleton of mature skeletal muscle. Proc Natl Acad Sci USA. 2016;113(39):10992-10997.

36. Han R, et al. Basal lamina strengthens cell membrane integrity via the laminin G domain-binding motif of alpha-dystroglycan Proc Natl Acad Sci USA. 2009;106(31):12573-12579.

37. Townsend D, Blankinship MJ, Allen JM, Gregorevic P, Chamberlain JS, Metzger JM. Systemic administration of micro-dystrophin restores cardiac geometry and prevents dobutamine-induced cardiac pump failure. Mol Ther. 2007;15(6):1086-1092.

38. Tinsley JM, Potter AC, Phelps SR, Fisher R, Trickett JI, Davies KE. Amelioration of the dystrophic phenotype of mdx mice using a truncated utrophin transgene. Nature. 1996;384(6607):349-353.

39. Peter AK, et al. Myogenic Akt signaling upregulates the utrophin-glycoprotein complex and promotes sarcolemma stability in muscular dystrophy. Hum Mol Genet. 2009;18(2):318-327.

40. Bies RD, et al. A 5' dystrophin duplication mutation causes membrane deficiency of alpha-dystroglycan in a family with X-linked cardiomyopathy. J Mol Cell Cardiol. 1997;29(12):3175-3188.

41. Sharpe KM, Premsukh MD, Townsend D. Alterations of dystrophin-associated glycoproteins in the heart lacking dystrophin or 
dystrophin and utrophin. J Muscle Res Cell Motil. 2013;34(5-6):395-405.

42. Michele DE, Kabaeva Z, Davis SL, Weiss RM, Campbell KP. Dystroglycan matrix receptor function in cardiac myocytes is important for limiting activity-induced myocardial damage. Circ Res. 2009;105(10):984-993.

43. Yasuda S, Townsend D, Michele DE, Favre EG, Day SM, Metzger JM. Dystrophic heart failure blocked by membrane sealant poloxamer. Nature. 2005;436(7053):1025-1029.

44. Townsend D, et al. Chronic administration of membrane sealant prevents severe cardiac injury and ventricular dilatation in dystrophic dogs. J Clin Invest. 2010;120(4):1140-1150.

45. Townsend D, Yasuda S, McNally E, Metzger JM. Distinct pathophysiological mechanisms of cardiomyopathy in hearts lacking dystrophin or the sarcoglycan complex. FASEB J. 2011;25(9):3106-3114.

46. Markham LW, et al. Abnormalities of diastolic function precede dilated cardiomyopathy associated with Duchenne muscular dystrophy. J Am Soc Echocardiogr. 2006;19(7):865-871.

47. Li Y, et al. Blunted cardiac beta-adrenergic response as an early indication of cardiac dysfunction in Duchenne muscular dystrophy. Cardiovasc Res. 2014;103(1):60-71.

48. Crawford GE, Faulkner JA, Crosbie RH, Campbell KP, Froehner SC, Chamberlain JS. Assembly of the dystrophin-associated protein complex does not require the dystrophin COOH-terminal domain. J Cell Biol. 2000;150(6):1399-1410.

49. Peter AK, Miller G, Crosbie RH. Disrupted mechanical stability of the dystrophin-glycoprotein complex causes severe muscular dystrophy in sarcospan transgenic mice. J Cell Sci. 2007;120(Pt 6):996-1008.

50. Harper SQ, et al. Modular flexibility of dystrophin: implications for gene therapy of Duchenne muscular dystrophy. Nat Med. $2002 ; 8(3): 253-261$.

51. Blankinship MJ, et al. Efficient transduction of skeletal muscle using vectors based on adeno-associated virus serotype 6 . Mol Ther. 2004;10(4):671-678.

52. Gregorevic P, Blankinship MJ, Allen JM, Chamberlain JS. Systemic microdystrophin gene delivery improves skeletal muscle structure and function in old dystrophic mdx mice. Mol Ther. 2008;16(4):657-664.

53. Fortunato MJ, et al. Development of rabbit monoclonal antibodies for detection of alpha-dystroglycan in normal and dystrophic tissue. PLoS One. 2014;9(5):e97567.

54. Hatzistergos KE, et al. S-Nitrosoglutathione Reductase Deficiency Enhances the Proliferative Expansion of Adult Heart Progenitors and Myocytes Post Myocardial Infarction. J Am Heart Assoc. 2015;4(7):e001974

55. Martins AS, et al. In Vivo Analysis of Troponin C Knock-In (A8V) Mice: Evidence that TNNC1 Is a Hypertrophic Cardiomyopathy Susceptibility Gene. Circ Cardiovasc Genet. 2015;8(5):653-664. 